\title{
Motives and Laterality: Exploring the Links
}

\section{Oliver C. Schultheiss ${ }^{1}$. Olivia S. Schwemmer ${ }^{1} \cdot$ Ksenia Khalaidovski $^{1}$}

Received: 19 October 2020 / Revised: 25 February 2021 / Accepted: 1 March 2021 /

Published online: 30 March 2021

(C) The Author(s) 2021

\begin{abstract}
Objectives We explored associations between the needs for power, achievement, and affiliation and functional cerebral asymmetries (FCAs), guided by three established hypotheses about the nature of these associations.

Methods One-hundred-and-seven participants completed picture-story measures of dispositional motives and activity inhibition (AI), a frequent moderator of motive-behavior associations, tasks measuring FCAs (line bisection, chimeric emotional face judgments, turning bias, perceptual and response asymmetries on the Poffenberger task), self-reported laterality preferences (handedness, footedness, ear and eye preference), and interhemispheric interaction (crossed-uncrossed difference). They also completed an experiment manipulating hand contractions (left, right, both, neither) while they worked on a second picture-story motive measure.
\end{abstract}

Results Dispositional power motivation was associated with stronger rightward asymmetry and less interhemispheric transfer in high-AI and stronger leftward asymmetry and more interhemispheric transfer in low-AI individuals. For the affiliation motive, findings were fewer and in the opposite direction of those for the power motive. These findings emerged for men, but not for women. Left- or right-hand contractions led to increases in power and achievement motivation, but not affiliation motivation. Only left-hand contractions led to decreased AI.

Conclusions We discuss these findings in the context of sex-dimorphic organizing and activating effects of steroids on motives and laterality.

Keywords Functional hemispheric asymmetry $\cdot$ Motivation · Motive dispositions · Sex difference $\cdot$ Power $\cdot$ Achievement $\cdot$ Affiliation $\cdot$ Picture-story exercise $\cdot$ Activity inhibition

Oliver C. Schultheiss

oliver.schultheiss@fau.de

1 Department of Psychology, Friedrich-Alexander University, Erlangen-Nürnberg,

Nägelsbachstraße 49b, 91052 Erlangen, Germany 
Motivational needs - or motives - represent capacities to experience strong affect from contact with certain classes of incentives (Atkinson, 1957; Schultheiss \& Köllner, 2021). In the case of the need for power (abbreviated nPower), the incentive is having impact on others (Winter, 1973); in the case of the need for achievement (nAchievement), the incentive is mastering a challenging task autonomously (McClelland et al., 1953); and in the case of the need for Affiliation (nAffiliation), the incentive is harmonious social contact (Koestner \& McClelland, 1992). Although these motivational needs do not represent an exhaustive list of fundamental motivational systems, 70 years of research on them has documented their close links with endocrine, cardiovascular, immune, and behavioral outcomes (McClelland, 1987, 1989; Schultheiss \& Köllner, 2021). Moreover, the measures employed to assess these motives are among the few in personality psychology featuring causal validity (Borsboom et al., 2004); that is, they reflect the effect of variations in motivational arousal (McClelland, 1958; Winter, 1998). Motives are assessed through content-coding of stories individuals write in response to pictures of social situations (picture story exercise, or PSE; McClelland et al., 1989; Schultheiss \& Pang, 2007).

Although other research has provided substantial evidence for the lateralization of perceptual, cognitive, affective-emotional, and motor functions (for overviews, Cutting, 1997; Gainotti, 2012; Ocklenburg \& Güntürkün, 2018), so far there has been little systematic inquiry into possible associations between motives and laterality. The present research aims to do this, both by exploring associations between dispositional motive measures and measures of functional cerebral asymmetries (FCA; e.g., Hausmann \& Güntürkün, 2000) - that is, processing advantages of one hemisphere over the other for a given function - and by examining effects of lateralized motor activation on motivational changes.

\section{McClelland's (1986) Right-Hemisphere Hypothesis}

But what kind of a relationship should one expect to observe between motives and the two halves of the brain? To our knowledge, McClelland (1986) was the first researcher working within the PSE motive measure paradigm to speculate about this issue. In trying to come to grips with why nAffiliation as assessed with the PSE had so little overlap with questionnaire measures of love and social closeness (see Köllner \& Schultheiss, 2014), but predicted physiological and behavioral indicators of affiliation rather well, he argued that nAffiliation and other motives are rooted in the right hemisphere $(\mathrm{RH})$ of the brain, whereas verbal judgments of one's motivational states or goals are mediated by the left hemisphere's (LH) propensity for weaving consistent stories about one's behavior in the absence of introspective access to the generators of behavior (Gazzaniga, 1985). More generally, McClelland (1986) posited that motives are RH phenomena and should therefore be associated with indicators of RH function, but not LH function. This claim resonates with a large body of research documenting the RH's role in emotional processing (see, for instance, Gainotti, 2012; Hellige, 1993; McGilchrist, 2009). This appears to be particularly true of deep 
and differentiated emotionality, whereas the LH seems to be associated with a more superficial, upbeat, Pollyannaish emotional style (McGilchrist, 2009), but also specifically with defensiveness and anger (Kuper et al., 2019). McClelland's claim also fits the observation that the $\mathrm{RH}$ is particularly involved in autonomic arousal processes characteristic of emotion and motivation and encompassing cardiovascular, endocrine, and other physiological outputs (Dimond \& Farrington, 1977; Wittling, 1995). The only test of his RH hypothesis that we are aware of is a recent study by Schütz and Schultheiss (2020) involving 67 gymnasts. The authors observed correlation patterns of nAchievement and nAffiliation, but not nPower, with a measure of perceptual laterality - the chimeric faces task - that were consistent with the RH hypothesis. No such evidence emerged for turning bias, a measure of motor laterality. In summary, the evidence in favor of McClelland's RH hypothesis is based on one small sample and has yielded inconsistent results across different motives and tasks.

\section{Kuhl and Kazen's (2008) Power/LH, Affiliation/RH Hypothesis}

Kuhl and Kazen (2008) provided a different perspective by hypothesizing that nPower, due to its orientation towards doing and having an effect on the world, is tied to the LH, which supports goal-directed, controlled action. In contrast, nAffiliation, due to its orientation towards being with others, is thought to be represented in the RH, which supports holistic and context-oriented forms of cognition. nAchievement is assumed to act as an interhemispheric integrator and therefore not to be lateralized in its own right. Kuhl and Kazen tested these ideas with a dot-probe task and verbal and pictorial stimuli associated with power, achievement, or affiliation being presented either in the left visual field (LVF) or the right visual field (RVF), followed by a dot in one or the other location. Consistent with their hypotheses, Kuhl and Kazen observed faster responses to dots replacing power stimuli presented in the RVF, and thus to the LH, than in the LVF, and thus to the RH. Conversely, participants responded faster to dots when they followed affiliation stimuli presented in the LVF than when they were presented in the RVF. Responses to achievement stimuli were more similar to those to power stimuli than to those to affiliation stimuli. Kuhl and Kazen's findings were based on a small sample size (combined $N=64$ across all three dot-probe studies). More importantly, while they document FCAs in response to motivational stimuli, they do not provide direct evidence that motives are lateralized in the suggested manner. This would have required the demonstration of significant Motive Disposition x Stimulus Type x Visual Field interactions, which the authors did not report.

\section{Schultheiss's (2018) AI-Moderation Hypothesis}

Building on work by McGilchrist (2009), who held that the hemispheres do not so much differ in what they do but in how they do it, Schultheiss (2018) suggested a third possibility, namely that motives may not be lateralized per se but that their 
behavioral manifestations may differ depending on which hemisphere is more involved in their expression. Reviewing the available literature on nPower and nAffiliation, he suggested that if these motives are expressed predominantly through LH-related functions, they tend to be more egoistically oriented towards dominance over others and relationship safeguarding, respectively, whereas if they are expressed via $\mathrm{RH}$-related functions, they tend to manifest themselves in more context-sensitive and adaptive social behavior (for review of hemispheric differences in cognitive and social functions, McGilchrist, 2009). Moreover, Schultheiss (2018) argued that activity inhibition (AI), a frequent moderator of motive effects on behavior in past research, is critical for whether motives are predominantly expressed via LH or RH functions. AI is measured by counting the frequency of the negation not (including its abbreviated forms; e.g., isn't) in PSE stories and has been shown to predict higher attentional sensitivity to stimuli presented in the LVF relative to stimuli presented in the RVF, particularly in emotionally engaging situations (Schultheiss et al., 2009). According to Schultheiss (2018), high AI makes motives more likely to engage the $\mathrm{RH}$, whereas low AI makes them more likely to become expressed through the LH. As a case in point, he refers to robust evidence showing that high nPower, combined with high AI, is associated with success in managers, both by objective measures of management performance and by the subjective judgment of their teams. In contrast, managers characterized by high nPower, but low AI, are more self-centered, less effective, and less well liked by their subordinates, which may reflect their tendency towards self-aggrandizing rather than socially adaptive behavior (e.g., Steinmann et al., 2015; for review, McClelland \& Burnham, 2003). Table 1 provides these and other examples of AI's moderating effect on motive-outcome associations. Notably, AI's moderating effect has been most frequently documented for nPower, sometimes for nAffiliation, and virtually never for nAchievement.

The validity of the idea that AI may represent a marker of motive-related FCAs has gained additional support through recent studies linking both nPower and AI to bone-growth markers of organizing effects of gonadal steroids that also affect brain development and FCAs (for review, Köllner et al., 2019; Schultheiss \& Köllner, 2021). Schultheiss et al. (2019) observed a robust association between digit ratio, a measure of prenatal exposure to gonadal steroids, and the confluence of nPower and AI, albeit in a sex-dimorphic manner. ${ }^{1}$ Likewise, Köllner and Bleck (2020) found a sex-dimorphic interaction between nPower and AI to be associated with the ulna/ fibula ratio, a putative measure of pubertal steroid exposure. Notably, in both studies the joint effect of nPower and AI was found for lateralized measures of bone growth; that is, the difference between the left and the right limb. Because these findings link the nPower $\mathrm{x}$ AI effect to asymmetric patterns of bodily growth, they provide additional, albeit indirect, support for the notion that AI may be intimately linked to functional asymmetries of the brain that channel the expression of nPower (and perhaps also other motives).

\footnotetext{
${ }^{1}$ Sex $x$ Laterality effects are pervasive in the neuropsychological literature. For instance, Lezak et al. (2012) state: "Compounding much of the data on sex differences in cognitive abilities is the effect of handedness, as left-handed males tend to perform more like right-handed females in showing superiority on tests of verbal skills and sequential processing, while left-handed females and right-handed males appear to have an advantage on visuospatial tasks [...] and for nonverbal auditory stimuli [...]." (p. 364).
} 


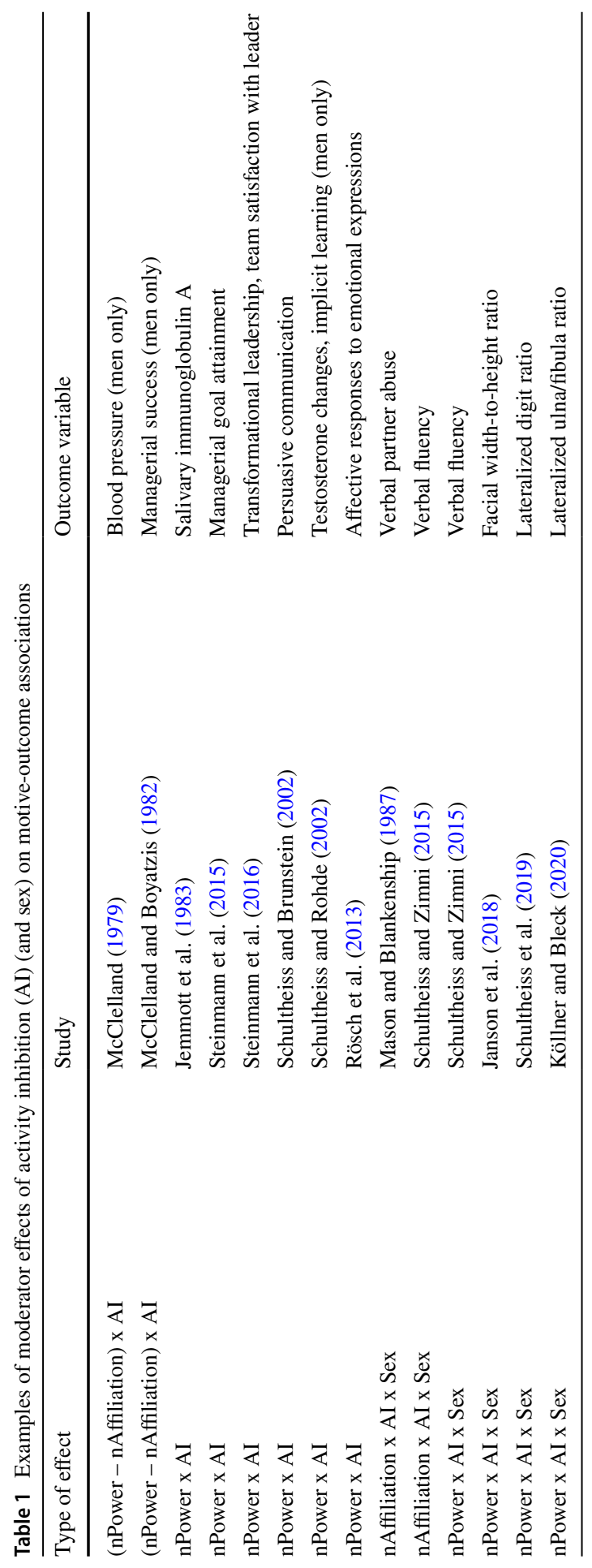


To summarize, although direct evidence from studies using FCA measures is lacking so far for Schultheiss's (2018) hypothesis, we believe his proposition to have more empirical support - albeit indirect, via AI interaction effects - than either McClelland's (1986) or Kuhl and Kazen's (2008) hypotheses. Given the general dearth of studies looking at the association between motives and FCAs, however, we kept an open mind with regard to all three hypotheses in our present research.

\section{The Present Study}

To arrive at a better understanding of how motives may be related to laterality, we followed a two-pronged approach. First, we examined associations between motive dispositions and FCA measures of perceptual and motor processes (see Table 2 for an overview). Measures of perceptual FCAs included stated preferences for eye and ear use, the chimeric faces task (Innes et al., 2016; Levy et al., 1983), the line bisection task (Jewell \& McCourt, 2000), and perceptual asymmetry scores on the Poffenberger task (Poffenberger, 1912). Measures of motor FCAs were stated hand and foot preference, observed turning bias (Yazgan et al., 1996), and motor asymmetry scores on the Poffenberger task. The Poffenberger task also yielded additional scores for crossed-uncrossed differences (CUD), a measure of relative time it takes to process perceptual input and produce a motor response to it ipsilaterally versus contralaterally (e.g., Saron et al., 2003), as well as directional measures quantifying the cost of transferring perceptual information from the $\mathrm{LH}$ to the $\mathrm{RH}$ and from the $\mathrm{RH}$ to the $\mathrm{LH}$ for initiating a motor response (e.g., Marzi et al., 1991; Thomas et al., 2018). Analyses for these combined perceptual/ motor integration indices were exploratory, because neither CUD, nor directional transfer scores represent direct FCA measures.

Table 2 Overview of functional cerebral asymmetries and the measures used to assess them in the present study (based on McGilchrist, 2009; McManus et al., 1999; Ocklenburg \& Güntürkün, 2018)

\begin{tabular}{|c|c|c|c|}
\hline Function & Left hemisphere & Right hemisphere & Measure \\
\hline Attention & $\begin{array}{l}\text { Focal, goal-oriented, } \\
\text { right visual field }\end{array}$ & $\begin{array}{l}\text { Broad, context-oriented, } \\
\text { both visual fields, bias } \\
\text { for left visual field }\end{array}$ & $\begin{array}{l}\text { Line bisection task } \\
\text { Perceptual asymmetry } \\
\text { (Poffenberger) }\end{array}$ \\
\hline Emotion processing & Non-dominant & Dominant & Chimeric faces task \\
\hline Language processing & Dominant & Non-dominant & Ear preference \\
\hline Vision & $\begin{array}{l}\text { No overall lateralization, } \\
\text { right eye for peeking }\end{array}$ & but preference for use of & Eye preference \\
\hline Motor output control & Dominant & Non-dominant & $\begin{array}{l}\text { Hand preference } \\
\text { Foot preference } \\
\text { Turning bias, motor } \\
\text { asymmetry (Poffen- } \\
\text { berger) }\end{array}$ \\
\hline $\begin{array}{l}\text { Transfer to contralateral } \\
\text { hemisphere }\end{array}$ & Low & High & $\begin{array}{l}\text { Directional crossed- } \\
\text { uncrossed difference } \\
\text { scores }\end{array}$ \\
\hline
\end{tabular}


If McClelland's (1986) RH hypothesis is correct, we expected all three motives to be consistently associated with evidence of preferred, better, or faster RH than LH processing. ${ }^{2}$ If Kuhl and Kazen's (2008) power/LH, affiliation/RH hypothesis is correct, we expected nPower to be associated with indications of better $\mathrm{LH}$ than $\mathrm{RH}$ processing, nAffiliation to be associated with the reverse, and nAchievement showing neither bias. Finally, we focused our analyses of Schultheiss's (2018) AI-moderation hypothesis more generally on nPower x AI effects, because these have been reported most frequently in the literature, and specifically on the exploration of sex-dimorphic nPower $\mathrm{x}$ AI effects, because these have been consistently reported for morphological measures (Janson et al., 2018; Köllner \& Bleck, 2020; Schultheiss et al., 2019) that present a window into steroid exposure of body and brain during critical developmental windows (Köllner et al., 2019). In general, we expected variations in nPower to predict indicators of more rightward FCAs in high-AI individuals and more leftward FCAs in low-AI individuals. However, we tentatively expected this to be the case predominantly for men, given the sexual dimorphism consistently observed in studies on morphological markers (see Table 1) and some findings on other outcomes even pointing in an opposite direction of this pattern for women (e.g., Schultheiss et al., 2019).

The second approach we employed in this research exploits the property of PSE-based motive measures to be sensitive to situationally induced changes in motivational states (McClelland, 1958; Winter, 1998). For instance, it has been shown that nPower increases in the context of a stressful public-speaking task (Wiemers et al., 2015), that nAffilation increases in response to exposure to affiliative movies (Schultheiss et al., 2004), and that nAchievement increases when performance is evaluated (Hofer et al., 2010). In the present study, we explored effects of differential hemispheric motor cortex activation on changes in motive and AI scores by having participants vigorously and rhythmically squeeze rubber balls with the left hand, the right hand, both hands, or neither hand while they were watching PSE picture cues and comparing the stories they subsequently wrote. In doing so, we were following an experimental protocol for testing lateralized spreading-activation accounts established in previous research using EEG measures of cortical arousal (Cross-Villasana et al., 2016; Harmon-Jones, 2006) and behavioral measures (e.g. Beckmann et al., 2013; Schiff et al., 1998), including story-telling tests similar to the PSE (Schiff \& Lamon, 1994), as criterion variables.

\footnotetext{
2 Theories of motivation generally assume that motives influence social behavior and task performance only in the presence of suitable situational incentives, representing a motive $\mathrm{x}$ incentive interaction. However, this does not preclude that motives can also have direct effects on basic neurocognitive processes (i.e., endophenotypes) such as those we tried to capture in the present research. Indeed, the reviewed findings relating $\mathrm{nPower} \mathrm{x} \mathrm{AI}$ effects to morphological measures suggest that brain organization may have been biased in specific ways due to the hormonal milieu during sensitive developmental phases, giving rise to subtle differences in cognitive processes. This is illustrated by Schultheiss and Zimni's (2015) finding that nPower predicted performance on a mental rotation task that featured no discernible power incentives.
} 
Putting these ideas together, we expected the following hand contraction effects: If McClelland's (1986) RH hypothesis holds, we would expect to see an increase in PSE scores for all three motives when the left hand, but not when the right hand squeezes the ball. If Kuhl and Kazen's (2008) power/LH, affiliation/RH hypothesis holds, we would expect to see nPower to increase if the ball is squeezed with the right hand and nAffiliation to increase if the ball is squeezed with the left hand. Schultheiss's (2018) AI-moderation hypothesis is more difficult to test with this paradigm, because it would require the observation of changes in the pattern (i.e., change interaction) of nPower and AI as dependent variable, controlling for the dispositional pattern (=basal interaction) of these variables. We therefore decided to tackle Schultheiss's hypothesis in a simplified manner by examining the effects of hemispheric motor activation on AI and motive measures separately, keeping in mind that this testing strategy may not reveal more complex changes in AI x nPower patterns.

\section{Method}

\section{Participants}

One-hundred-and-seven participants (64 women, 43 men; mean age 24.94 years, $S D=4.28$ ) were tested between July and October 2018 at Friedrich-Alexander University, Erlangen, Germany, for a study advertised under the title of "Perception, action, and story-telling". The procedures used in this study were approved by the local ethics committee. Participants provided written informed consent before testing started and received $€ 15$ remuneration after completion of the testing session. A sample size $>100$ participants was targeted to obtain $80 \%$ power for reliably detecting bivariate correlations of 0.27 or better; that is, effect sizes that are typical of associations between motives (or AI) and cognitive measures (e.g., Schultheiss et al., 2009; 2011; Schütz \& Schultheiss, 2020). For two-way interactions (e.g., Sex $\mathrm{x}$ Motive or Motive x AI), power is $<70 \%$ (based on the differences of motive/laterality slopes reported in Köllner \& Bleck, 2020, p. 107), and power is still lower for three-way interactions (e.g., Sex x Motive x AI).

\section{Design}

The study had both correlational and experimental features. The correlational design aspect pertained to analyses of the association between dispositional motive and AI levels, corrected for PSE story length, as well as sex with measures of perceptual and motor FCAs and measures of interhemispheric transfer. The experimental design aspect pertained to the analysis of the effects of unilateral/bilateral motor activation on changes in motive and AI scores on the PSE. Specifically, we used a 2 (left hand contraction vs no contraction) $\times 2$ (right hand contraction vs no contraction) ANCOVA design, with dispositional motive and AI scores as covariates and post-manipulation motive and AI scores as dependent variables. 


\section{Measures}

\section{Dispositional Motives and AI}

To assess participants' dispositional motives and AI, we administered a computer-based PSE using standard instructions and featuring the pictures trapeze artists, couple by river, soccer duel, and men on ship deck described in Schultheiss and Pang (2007, Table 1). Pictures were chosen so that they provided a broadband measure of nAffiliation, nAchievement, and nPower, with an expected mean score of at least 3 for each motive. Participants saw each picture in randomized order for $10 \mathrm{~s}$ and were then given 4 min to write a story into a composition window. After $4 \mathrm{~min}$, they were instructed to finish the present story and move on to the next picture-story by clicking a button.

Stories were later coded, following Winter's (1994) manual, for motivational imagery by a trained coder who was blind to participants' condition assignment and results on other tests. In brief, power imagery is coded when someone shows a concern for having impact through (1) strong, forceful actions, (2) controlling or manipulating others, (3) influencing, arguing with, or persuading others, (4) providing unsolicited help or advice to others, (5) impressing others or showing a concern with fame or prestige, or (6) eliciting strong emotions in others. Achievement imagery is coded for (1) adjectives suggesting good performance, (2) goals or performances that are portrayed in a positive way, (3) competing with someone or winning a competition, (4) failure leading to negative affect, and (5) unique accomplishments. Affiliation imagery is coded for (1) positive affect expressed in the context of a relationship, (2) sadness about relationship disruption or loss, (3) companionate activities, and (4) nurturant help and assistance. Following the recommendations of Schönbrodt et al. (2020), the coder ignored Winter's (1994) second-sentence coding rule and instead coded every instance of motivational imagery. The coder had previously exceeded $85 \%$ inter-scorer agreement on calibration materials contained in the manual.

AI (frequency of the German word for the negation not, "nicht") and story word counts were determined, and motive imagery coding was aided, by the PSECoder (Frisch \& Schultheiss, 2012; http://www.psych2.phil.uni-erlangen.de/\% 7Eoschult/humanlab/resources/resources_PSECoder.htm).

Dispositional nPower $(M=3.23, S D=1.93)$, nAchievement $(M=6.76$, $S D=3.23)$, and nAffiliation $(M=5.93, S D=2.91)$ as well as AI raw scores $(M=2.67, S D=2.37)$ were not normally distributed according to the Shapiro-Wilk test, $p \mathrm{~s}<0.006$. To correct for skew, we subjected motive and AI scores to a square-root transformation after adding a constant of 1 . We then converted motive and AI scores to $\mathrm{z}$ scores after regressing total word count $(M=412$, $S D=122$ ) from motive and AI scores to remove the shared variance with narrative fluency ( $r \mathrm{~s}$ with transformed motive and AI scores $>0.40, p \mathrm{~s}<0.001$ ). The motive and AI scores resulting from these procedures were independent of PSE protocol length, did not significantly differ from a normal distribution, Shapiro Wilk $p s>0.30$, and were used in all further analyses of associations between dispositional PSE measures and FCAs. Because one participant did not follow 
instructions and failed to provide stories that could be scored for motive imagery, PSE data are available for 106 participants.

\section{Experimentally Induced Motive and Al Changes}

To implement experimental conditions and assess their effect on changes in motives and AI, we administered and coded a second 4-picture PSE. All methodological aspects were identical to the first, except for the following:

First, we used the pictures tango, bicycle race, girlfriends in café with male approaching, and ship captain to again get a broadband measure of all three motives and AI, with an expected mean of at least 3 for each motive. The first picture was pretested in an unpublished pilot study (Lang, 2016) and depicts a man and a woman dancing a tango on a stage. The other three pictures are described in Schultheiss and Pang (2007, Table 1).

Second, before writing each story, participants listened via headphones to a clicking sound presented at $92 \mathrm{bpm}$ for a total of $45 \mathrm{~s}$. During the first $35 \mathrm{~s}$ of sound presentation, the screen was black; during the last $10 \mathrm{~s}$, the PSE picture was shown. The sound ended as soon as the story-composition window was subsequently presented. Participants in the left-hand (right-hand) contraction condition were instructed to take a two-inch soft rubber ball into their left hand (right hand) and squeeze the ball in sync with the click sound for as long as the sound was presented. Participants in the both-hands contraction condition were given two identical balls and instructed to squeeze with both hands in the same manner. Participants in the no-contraction condition were asked to just listen to the sound and look at the picture.

Third, to model changes in motive and AI scores in the ANCOVAs reported below, we used untransformed raw scores from the baseline and the experimentalmanipulation PSEs. We thus used commensurate motive and AI baseline and postmanipulation measures and assumed that (a) story-length effects (retest $r=0.90$, $\mathrm{p}<0.001$ ) would be effectively controlled for by adjusting for baseline motive and AI measures (which were affected by story length, too) and (b) similar deviations from normal distributions would be present in both baseline and post-manipulation scores, resulting in normally distributed regression residuals (verified via Q-Q plots). In addition, we examined for all analyses reported below whether they substantially changed if either log-transformed scores were used for both baseline and post-manipulation scores, or if post-manipulation word count was added as an additional covariate ( $r \mathrm{~s}$ with raw motive and AI scores $>0.44, p s<0.001$ ), or if outliers were present and subsequently removed. The analyses reported below were robust for these additional checks. Scores were in the expected range for nPower $(M=5.65$, $S D=3.06)$, nAchievement $(M=6.84, S D=3.60)$, nAffiliation $(M=5.26, S D=2.85)$, $\mathrm{AI}(M=3.63, S D=2.65)$, and word count $(M=438, S D=129)$.

\section{Self-Reported Perceptual and Motor Laterality Preferences}

To assess participants' handedness, we administered the following questions: "Which hand do you use for handwriting?", "Which hand do you use for brushing your teeth?", and "Which hand do you use for holding the phone?" (response 
options: left hand, coded 1; sometimes one hand, sometimes the other, coded 0; or right hand, coded -1 ). The three items were positively correlated (Spearman $r s>0.30)$. We averaged them into a handedness scale.

To assess participants' footedness, we administered the following questions: "If you have to jump across a little moat, which foot do you use to push yourself off?" and "For a long jump, which foot do you use to push yourself off?" (response options: left foot, coded 1; sometimes one foot, sometimes the other, coded 0; or right foot, coded -1 ). Both items were positively correlated, Spearman $r=0.61$ ), and we averaged them into a footedness scale.

Eye and ear preference were assessed with one item each. For the former, it was "If you look through something with just one eye - a spyglass, for instance - which eye do you squeeze shut?", and for the latter, it was "To which ear do you hold your phone during a phone conversation?". Response options were left eye/ear (1), sometimes one, sometimes the other (0), and right eyelear $(-1)$. The response scale was reverse coded for eye use, because indicating which eye is shut implies the other eye being the preferred one.

\section{Turning Bias}

To assess participants' turning bias, we used Schütz and Schultheiss's (2020) adaptation of Yazgan et al.'s (1996) task. In brief, participants were required to walk up and down an empty hallway five times and turn around at centered markings on the floor at both ends. These markings were located $6.5 \mathrm{~m}$ apart. This yielded a total of 11 observable turns ( 6 at the back, 5 at the front of the hallway), recorded on video for later coding. The experimenter, who stood in the middle at one end of the hallway, told participants that this task was about their gait and instructed them to walk up and down freely at first, then with their left eye covered, then with their right eye covered, then with their left ear covered, then with their right ear covered, and finally with both ears covered. These instructions were intended to deflect participants' attention from the goal of the procedure; that is, the assessment of turning bias. All videos were later independently coded by two coders. Intercoder reliability was excellent, Pearson $r=0.99$. Because the second coder had recorded all observations correctly on paper, but made a mistake in one case transferring the data to the data file (otherwise $r$ would have been 1.00), only the codings of the first coder were used for further analyses. Participants' turning responses were consistent, as reflected by a strong positive correlation between the first and the second 5 turns, Pearson $r=0.87$.

\section{Chimeric Faces Task}

We used Schütz and Schultheiss's (2020) adaptation of the chimeric faces task (CFT). In brief, this CFT consists of 16 horizontally arranged pairs of chimeric faces, one showing a happy expression on the left and one showing a happy expression on the right side, with pairing (left/right versus right/left) balanced across trials. Randomized presentations of the 16 pairs were blocked twofold for a total of 32 trials. Participants were instructed to indicate by keypress which of the two variants 
depicted in each pair they judged as more emotional. Responses were recoded such that 1 indicated the choice of the emotionlneutral variant and 0 indicated the choice of the neutrallemotion variant. Based on the distribution of response latencies, we regarded responses with a latency $<500 \mathrm{~ms}$ as premature and invalid and therefore did not count them (1.43\% of all responses). Further scrutiny of the data revealed that the data of two participants consisted mostly of premature responses, were therefore invalid, and dropped from the CFT data (hence, $N=105$ for CFT data). Another two participants provided valid data only on the first block, but not on the second; data from the second block were therefore dropped in this case and total CFT scores were based on the first block only. Total CFT scores were calculated by dividing the sum of valid " 1 " responses by the sum of valid " 1 " plus " 0 " responses and multiplying the total with 100 . The resulting score reflects the percentage of emotionlneutral faces chosen and thus an $\mathrm{RH}$ bias for processing emotional faces. The test-retest Pearson correlation from the first to the second half was 0.64. Cronbach $\alpha$ for the full 32-item scale was 0.80 .

\section{Line Bisection Task}

We created a computer-based version of the line bisection task (LBT) by serially presenting lines of varying length $(21 \mathrm{~cm}$ vs $24 \mathrm{~cm})$ with their center in the left ( 8 trials), middle ( 8 trials), or right ( 8 trials) of the computer screen. In doing so, we translated the typical horizontal position variations of paper-and-pencil LBT versions to the computer and also allowed horizontal position to become a withinsubjects factor in subsequent analyses (previous results strongly suggest that within individuals LBT scores are affected by horizontal position; e.g., Hausmann et al., 2002). The vertical position also varied orthogonally to the horizontal position. The changing positions were intended to force participants to reorient themselves spatially on each trial. Participants were instructed to place a mark with the mouse on the middle of each line. Inspection of responses suggested that response latencies $<437 \mathrm{~ms}$ and responses $<110 \% \mathrm{l}$ (with $50 \%$ marking the midpoint of a line) represented premature and thus invalid responses $(0.12 \%$ of the data). These responses were set to missing. Because scores for the three horizontal positions differed markedly, $F(2,210)=264, p<0.001, \eta^{2}=0.72$, we used separate averaged scores for the left, the middle, and the right screen position in all subsequent analyses. Scores were calculated as percentage of the full line, with $50 \%$ marking the exact middle of a line and higher scores - that is, midpoints set to the left of the middle - reflecting increasing pseudoneglect and thus increasing $\mathrm{RH}$ dominance for spatial attention. Conversely, scores $<50 \%$ reflected increasing neglect and thus increasing LH dominance. Due to technical problems, only 103 participants completed the LBT. Cronbach $\alpha$ s for lines presented in the left, middle, and right sections of the screen were $0.80,0.78$, and 0.81 , respectively.

\section{Poffenberger Task}

We used Borchert's (2013) adaptation of the Poffenberger task described by Berlucchi et al. (1971). Participants were instructed to press a key as quickly as possible 
whenever they saw a dot appear to the left or right of a centered fixation cross on the screen. On each trial, participants were alerted to the imminent display of the dot through a beep presented via headphones and a brief flicker of the fixation cross. After practicing the task, participants worked on 120 trials resulting from a 2 (visual field: left, right) $\times 2$ (response hand: left, right) $\times 3$ (dot distance from fixation cross: near, medium, far) $\times 2$ (blocks) $\times 5$ (repetitions) factorial design. Invalid trials (response latencies $<160 \mathrm{~ms}$ or $>600 \mathrm{~ms} ; 10.05 \%$ ) were repeated until a valid response was recorded. Data from three participants were not recorded due to a programming error. Another five participants produced 50 or more error responses, and we therefore excluded them from all further analyses involving the Poffenberger task; hence $N=99$. Analyses using median response times revealed effects of visual field (left: $M=250 \mathrm{~ms}, S D=34 \mathrm{~ms}$; right: $M=247 \mathrm{~ms}, S D=31 \mathrm{~ms} ; F[1$, 98] $=5.52, p=0.021, \eta^{2}=0.053$ ), response hand (left: $M=247 \mathrm{~ms}, S D=30 \mathrm{~ms}$; right: $\left.M=250 \mathrm{~ms}, S D=35 \mathrm{~ms} ; F[1,98]=6.29, p=0.014, \eta^{2}=0.060\right)$, and a visual field $x$ response hand interaction (left hand/LVF: $M=246 \mathrm{~ms}, S D=32$; left hand/ RVF: $M=248 \mathrm{~ms}, S D=31$; right hand/LVF: $M=254 \mathrm{~ms}, S D=38$; right hand/RVF: $M=246 \mathrm{~ms}, S D=34 \mathrm{~ms} ; F[1,98]=19.76, p<0.0005, \eta^{2}=0.168$; see Fig. 1$)$. We therefore calculated (a) a perceptual laterality score by subtracting LVF latencies from RVF latencies, (b) a motor laterality score by subtracting left-hand latencies from right-hand latencies, (c) a crossed-uncrossed difference (CUD) score by subtracting averaged left-hand/LVF and right-hand/RVF latencies from averaged lefthand/RVF and right-hand/LVF latencies, and (d), as indices of directional interhemispheric transfer effects, a right-to-left transfer $(\mathrm{R}>\mathrm{LT})$ score and a left-to-right transfer $(\mathrm{L}>\mathrm{RT})$ score by subtracting average latencies in response to ipsilateral targets from average latencies in response to contralateral targets during trials requiring

Fig. 1 Interaction effect of response hand and visual field on response latency on the Poffenberger task. Figure available at https://osf.io/84usb/, under a CC-BY4.0 license

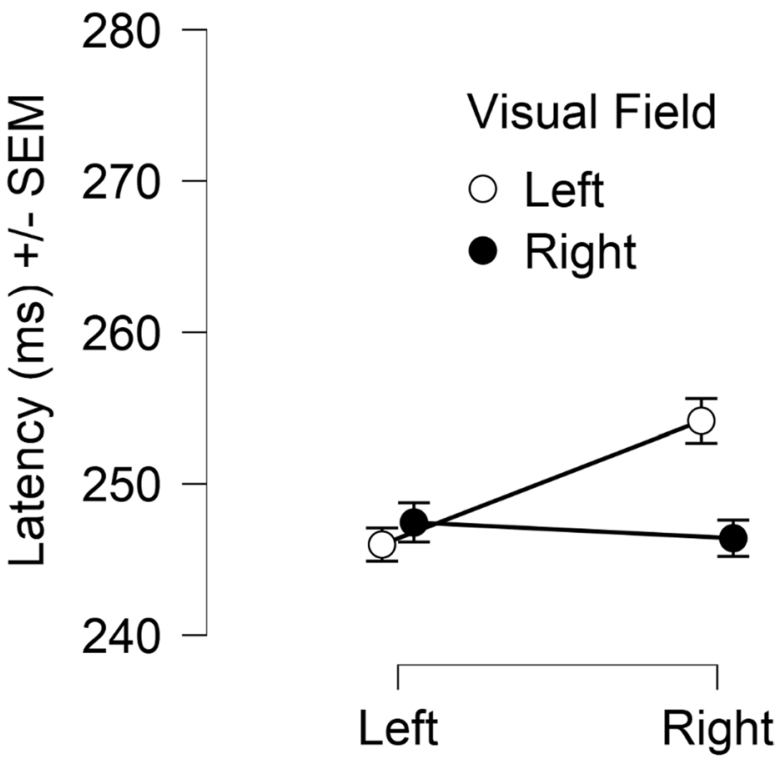

Hand 
a right-hand and a left-hand response, respectively (see Thomas et al., 2018). Higher values on the laterality scores represent relatively quicker RH-mediated responses for LVF and left hand; higher values on the CUD represent relatively slower interhemispheric than intrahemispheric processing, and higher values on $\mathrm{R}>\mathrm{LT}$ and L $>$ RT scores reflect relatively slower rates of information transfer from the RH to the $\mathrm{LH}$ and from the $\mathrm{LH}$ to the $\mathrm{RH}$, respectively.

\section{Procedure}

Upon arrival at the laboratory, participants first completed the turning bias assessment. They were then placed in single-participant cubicles and completed the CFT, the Poffenberger, the LBT, the dispositional PSE, the experimental-manipulation PSE, and a questionnaire about their perceptual and motor habits and preferences as well as some biographical background information on Windows PCs equipped with standard QWERTZ keyboards, mice, headphones, and 23" color monitors. Except for the turning bias task, all tasks were programmed and presented using Inquisit 5.011 (Millisecond Software, LLC; Seattle, Washington). All Inquisit task modules and an illustration of the turning bias task are available on https://osf.io/84usb/.

\section{Statistical Processing, Analyses, and Data Availability}

Data preprocessing was done in SYSTAT 13. All main analyses were run and graphs generated in JASP 13.1. All data and syntax files as well as all integrated JASP analysis files for reproducible data analyses are available from https://osf.io/84usb/. All $\eta^{2}$ represent partial effect sizes.

\section{Results}

\section{Descriptive Data and Correlations}

Table 3 provides descriptive statistics and correlations for the main study variables. CFT, LBT, and Poffenberger motor laterality all showed significant leftward biases, suggesting RH dominance for these tasks. In contrast, handedness, footedness, eye and ear preference as well as Poffenberger perceptual laterality were characterized by a significant rightward bias, suggesting LH dominance for these measures. Turning bias, by showing a behavioral leftward bias, can be considered to be an indicator of LH control, because the right half of the body initiates the turn to the left. CUD scores were significantly higher than zero, indicating the slower interhemispheric than intrahemispheric processing at the sample level typically observed (e.g., Marzi et al., 1991). Handedness was positively correlated with eye and ear preference, which also correlated positively with each other. Both turning bias and CFT had no reliable overlap with other FCA measures. Both left and right LBT scores correlated positively with mid-screen LBT scores, but not with each other, suggesting that lines initially presented to the LVF and the RVF, respectively, may assess separate 


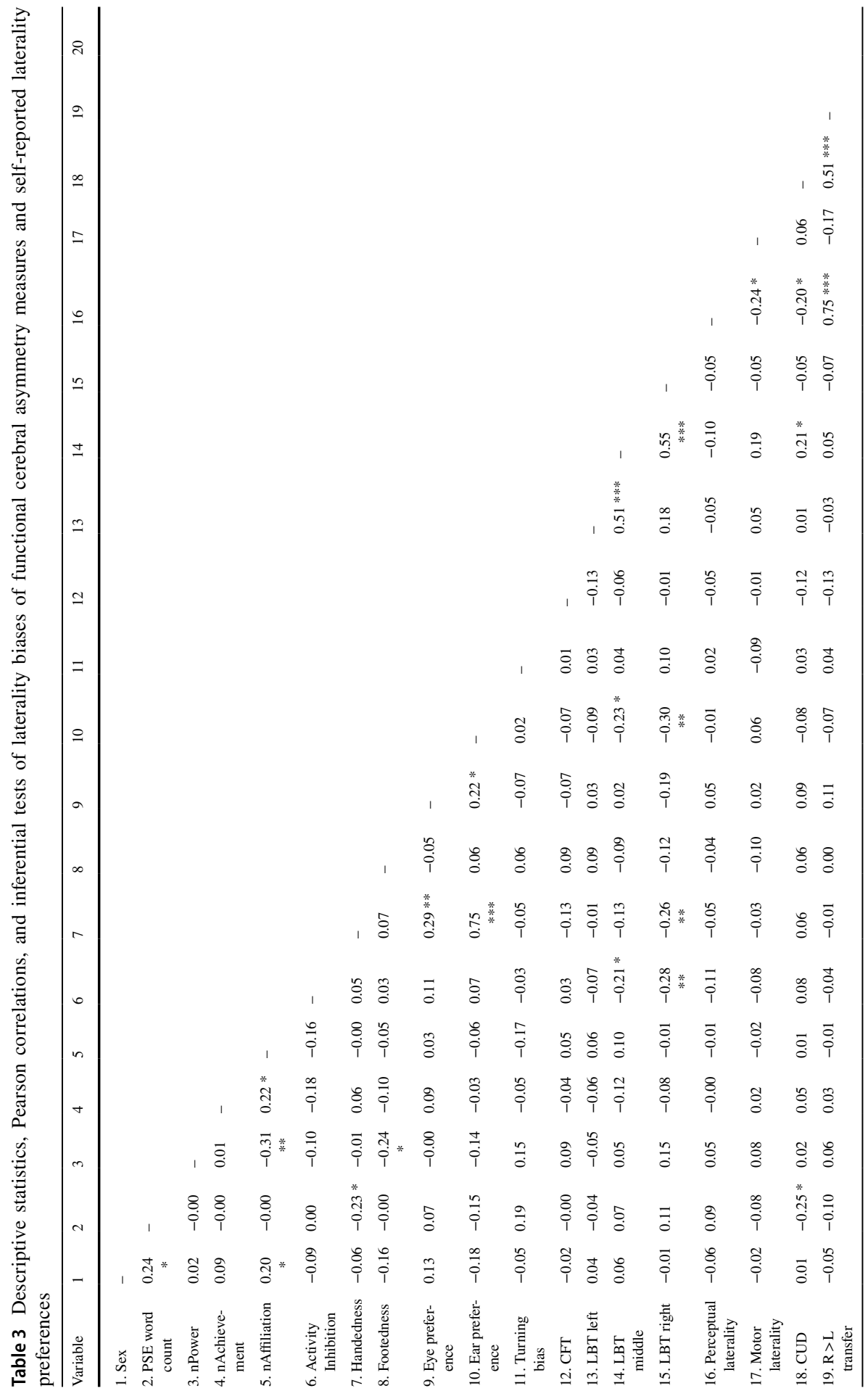




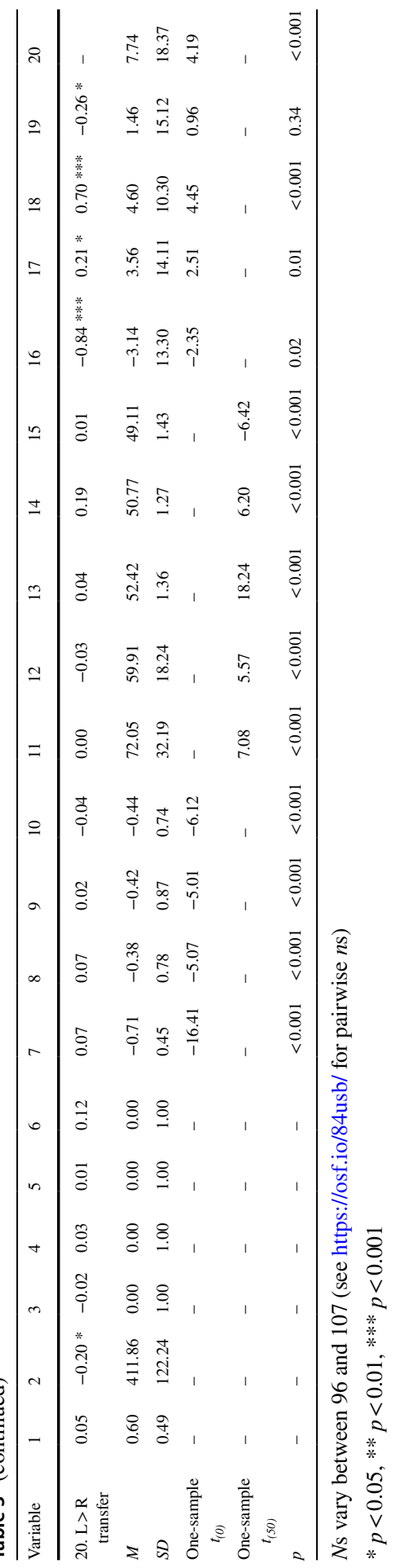


hemispheric attentional processes. Moreover, LBT scores frequently featured a pattern of "escalating" correlations, increasing in size from left to right. This pattern was most pronounced for their negative associations with handedness and ear preference: in each case, the LBT score obtained from the RVF showed the strongest correlation with these measures. Scores derived from the Poffenberger task showed the expected correlations with each other, but only little reliable overlap with other FCA measures. The only exception was the positive association between CUD scores and mid-screen LBT scores, indicating stronger pseudoneglect in individuals with relatively better intra- than interhemispheric processing. Sex was not significantly associated with any laterality measure or scores based on the Poffenberger task.

We next turn to measures derived from the PSE. Women wrote longer PSE stories and stories that contained more affiliation imagery than men, as has been documented in previous research (Drescher \& Schultheiss, 2016; Schultheiss et al., 2020). Moreover, participants who wrote longer stories were also more strongly right-handed, tended to show a stronger leftward turning bias $(p=0.05)$, and had lower CUD scores, suggesting better interhemispheric integration. The latter effect appeared to be particularly associated with faster left-to-right transfer of information. Higher nPower was associated with stronger right-footedness, nAchievement and nAffiliation were not reliably associated with any FCA or Poffenberger measure. AI showed the previously mentioned pattern of escalating correlations on the LBT, with higher AI scores being associated with significantly less pseudoneglect for lines presented in the RVF and, to a lesser extent, for lines presented in the middle of the screen.

\section{Correlational Analyses with Dispositional Measures}

\section{McClelland's (1986) Hypothesis}

Table 3 shows no consistent pattern of correlations that would suggest motives to be associated with stronger $\mathrm{RH}$ processing. When we combined all dispositional motive variables into one overall score, thus testing for associations of the shared variance of motive measures with laterality measures, we were likewise unable to find significant or consistent relationships. The only exception was footedness, which was negatively associated with the motive sum score, $r=-0.22, p=0.02$, a finding in the opposite direction of McClelland's hypothesis.

\section{Kuhl and Kazen's (2008) Hypothesis}

Although one would expect correlations of nAffiliation with laterality measures to be positive, representing the association between this motive and a $\mathrm{RH}$ processing advantage postulated by Kuhl and Kazen (2008), seven of the eleven correlations of nAffiliation with FCA measures presented in Table 3 have a negative sign. Similarly, although correlations between nPower and FCA measures would be expected to be negative and thus consistent with the postulated LH processing advantage, six of the eleven correlations have a positive sign. We also ran a series of regression analyses 
Fig. 2 Effects of sex, activity inhibition (split at tertiles), and nPower on mid-screen line bisection task scores, Poffenberger crossed-uncrossed scores, chimeric faces task scores, and laterality preference scores averaged across handedness, footedness, eye, and ear preference. For laterality measures, higher/ positive scores represent a behavioral leftward bias (right hemisphere), and lower/negative scores a behavioral rightward bias (left hemisphere). Figure available at https://osf.io/84usb/, under a CC-BY4.0 license

of all FCA measures on scores of nPower and nAffiliation simultaneously to explore whether the negative association between these motive measures suppresses more hypothesis-consistent statistical effects. However, none of these regressions revealed any significant associations between motives and FCA measures beyond those shown in Table 3 for zero-order correlations. In the 11 regressions we ran, the expected pattern of a negative slope for nPower and a positive slope for nAffiliation emerged only once, whereas the opposite pattern, suggesting a RH advantage for nPower and a LH advantage for nAffiliation, emerged three times (in the remainder of the analyses, both motives had either a positive or a negative slope). Inclusion of nAchievement did not lead to more clear-cut results regarding Kuhl and Kazen's hypothesis.

\section{Schultheiss's (2018) Hypothesis}

We regressed laterality and Poffenberger transfer measures on nPower, AI, sex and their two- and three-way interactions.

LBT For LBT, we found a Visual Field x nPower x AI x Sex interaction, F(1, $194)=3.82, p=0.024, \eta^{2}=0.038$, that we could trace back to a nPower x AI x Sex effect for the middle LBT score, $B=-0.795, S E=0.286, p=0.006, \Delta R^{2}=0.070$, and that did not emerge for either the left or the right LBT score, $p \mathrm{~s}>0.77$. As shown in Fig. 2, the effect was due to men whose nPower was negatively related to pseudoneglect, reflecting relatively more LH engagement in the task, when they were low in AI, but positively related, reflecting relatively more $\mathrm{RH}$ engagement, when they had medium or high AI; for the nPower x AI interaction, $B=0.838, S E=0.263$, $p=0.020, \Delta R^{2}=0.133$. In women, the nPower $\mathrm{x}$ AI effect on middle LBT scores was not significant, $B=-0.156, S E=0.135, p=0.25, \Delta R^{2}=0.020$.

Poffenberger Task We observed a significant Visual Field $\mathrm{x}$ Response Hand $\mathrm{x}$ nPower x AI x Sex interaction, $F(1,90)=6.77, p=0.011, \eta^{2}=0.070$, that could be simplified into a nPower $\mathrm{x}$ AI $\mathrm{x}$ Sex interaction on CUD scores, which captured the Visual Field $x$ Response Hand term, as dependent variable, $B=-6.81, S E=2.62$, $p=0.011, \Delta R^{2}=0.069$. As shown in Fig. 2, the interaction was due to men's nPower being negatively associated with CUD in low-AI individuals and positively in high-AI individuals; for the nPower x AI effect, $B=5.98, S E=2.22, p=0.011$, $\Delta R^{2}=0.175$. In women, the nPower $\mathrm{x} A \mathrm{I}$ interaction was not significant, $B=-0.826$, $S E=1.31, p=0.53, \Delta R^{2}=0.007$. We also observed a significant Visual Field $\mathrm{x}$ Response Hand $\mathrm{x}$ nAffiliation $\mathrm{x}$ AI x Sex interaction, $F(1,90)=5.01, p=0.028$, $\eta^{2}=0.053$, that could be simplified into a nAffiliation $\mathrm{x}$ AI $\mathrm{x}$ Sex interaction on 
Men

Women
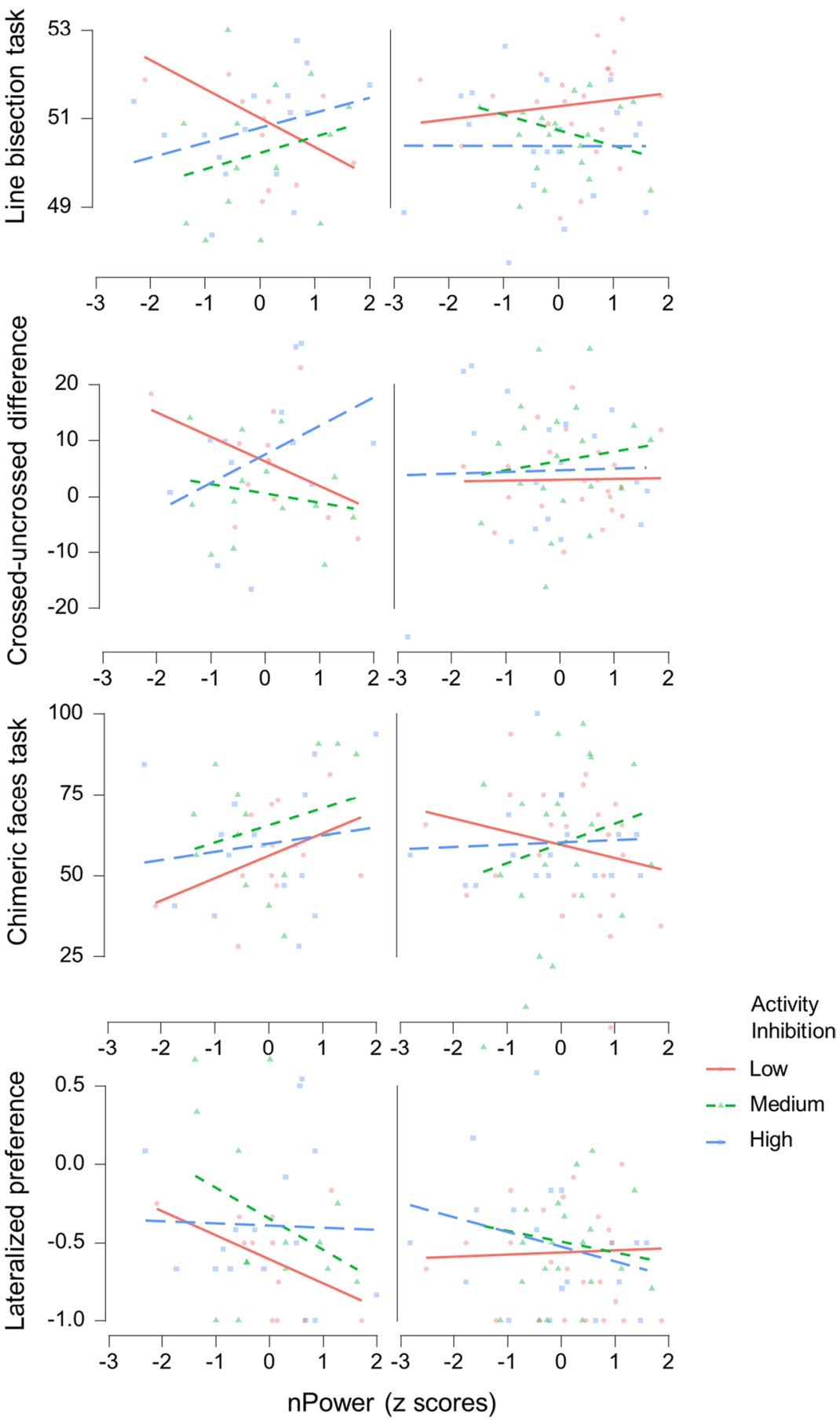
CUD scores, $B=6.21, S E=2.77, p=0.028, \Delta R^{2}=0.052$. As shown in Fig. 3 , the interaction was due to men's nAffiliation being positively associated with CUD in low-AI individuals and negatively in high-AI individuals; for the nAffiliation $\mathrm{x}$ AI effect, $B=-4.08, S E=1.93, p=0.041, \Delta R^{2}=0.116$. In women, the nAffiliation x AI interaction was not significant, $B=2.13, S E=2.00, p=0.29, \Delta R^{2}=0.020$.

CFT The nPower $\mathrm{x}$ AI x Sex interaction failed to become significant, $B=-6.18$, $S E=4.32, p=0.16, \Delta R^{2}=0.020$. As shown in Fig. 2, at the descriptive level nPower was associated with more RH-driven emotionality judgments in men, partial $r=0.24, p=0.13$, and in high-AI women; for the nPower $\mathrm{x} \mathrm{AI}$ interaction in women, $B=2.58, S E=2.12, p=0.25, \Delta R^{2}=0.023$.

Self-Reported Side Preferences For analyses involving handedness, footedness, as well as eye and ear side preferences, we modeled these variables as a within-subjects factor in our analyses. Three significant findings emerged. First, we found a nPower
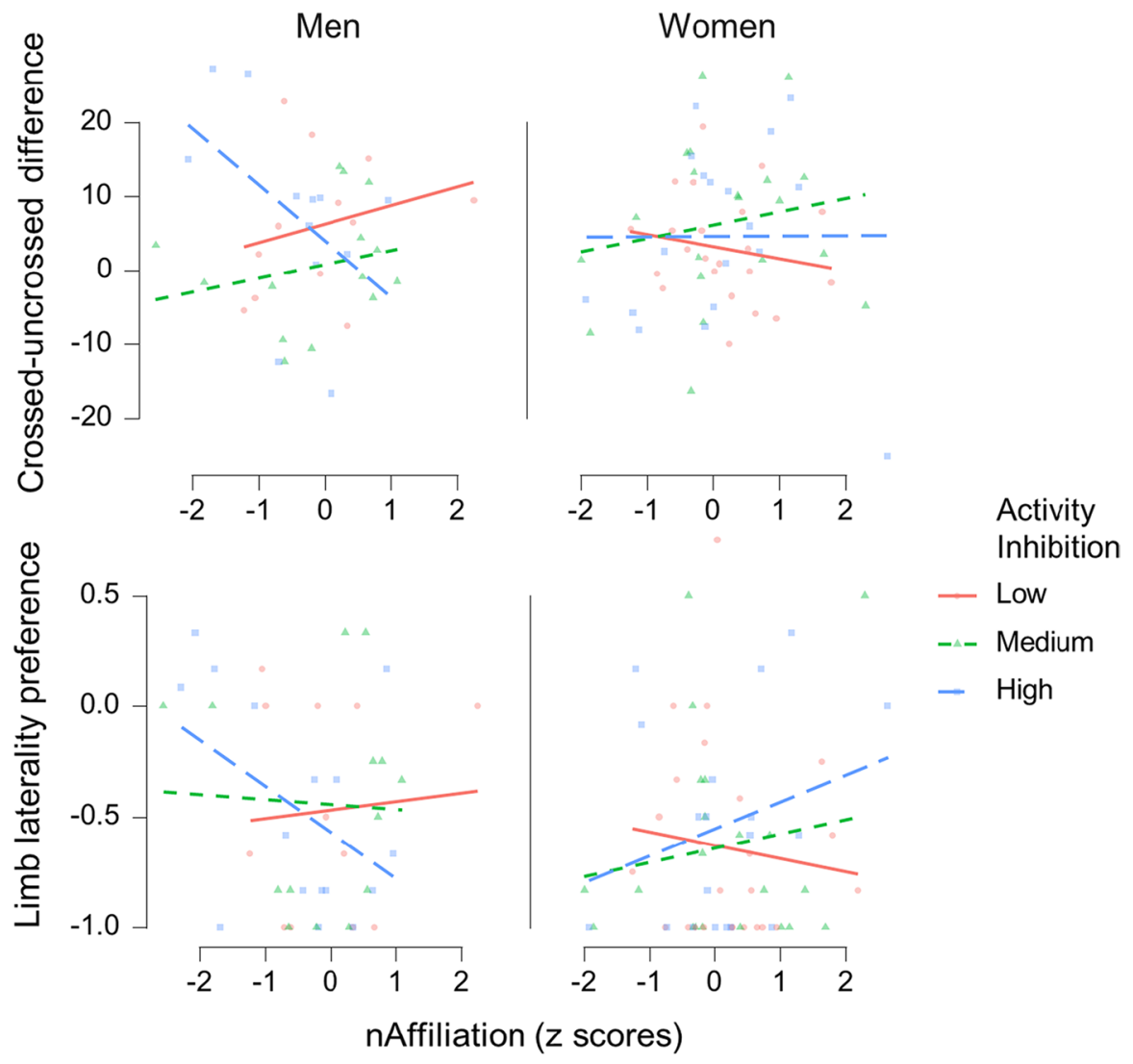

Fig. 3 Effects of sex, activity inhibition (split at tertiles), and nAffiliation on Poffenberger crosseduncrossed difference scores and limb laterality preferences (averaged handedness and footedness, higher scores represent a more leftward preference). Figure available at https://osf.io/84usb/, under a CC-BY4.0 license 
$\mathrm{x}$ AI x Sex interaction, $B=-0.222, S E=0.102, p=0.032, \Delta R^{2}=0.044$, that was not moderated by the within-subjects factor. As shown in Fig. 2, the interaction was due to men's nPower being associated with a greater preference for the right side of the body, and thus with greater LH engagement, in low-AI individuals and with no preference in high-AI individuals; for the nPower x AI effect, $B=0.196, S E=0.091$, $p=0.038, \Delta R^{2}=0.101$. In women, the nPower $\mathrm{x}$ AI interaction was not significant, $B=-0.027, S E=0.049, p=0.59, \Delta R^{2}=0.005$. Second, we also found a Measure $\mathrm{x}$ nAffiliation $\mathrm{x}$ AI $\mathrm{x}$ Sex interaction, $F(3,294)=3.17, p=0.025, \eta^{2}=0.031$, that could be traced back to a significant nAffiliation $\mathrm{x}$ AI $\mathrm{x}$ Sex interaction for averaged motor laterality preferences (i.e., handedness and footedness), $B=0.249, S E=0.104$, $p=0.019, \Delta R^{2}=0.052$, that did not emerge for averaged perceptual laterality (i.e., eye and ear preferences), $B=-0.032, S E=0.148, p=0.83, \Delta R^{2}<0.001$. As shown in Fig. 3, the interaction was due to men's nAffiliation being associated with a greater preference for the right side of the body, and thus with greater LH engagement, in high-AI individuals and with no preference in low-AI individuals; for the nAffiliation $\mathrm{x}$ AI effect, $B=-0.159, S E=0.070, p=0.030, \Delta R^{2}=0.114$. For women, the pattern of results was in the opposite direction, but failed to become significant, $B=0.090, S E=0.077, p=0.25, \Delta R^{2}=0.022$. Third, because the association of AI with laterality is of theoretical interest (see Schultheiss et al., 2009), we also report a significant Measure x AI x Sex interaction, $F(3,306)=3.64, p=0.013, \eta^{2}=0.034$, which was mainly due to two significant AI x Sex interaction effects in opposite directions for handedness, $B=-0.203, S E=0.090, p=0.026, \Delta R^{2}=0.048$, and footedness, $B=0.315, S E=0.155, p=0.046, \Delta R^{2}=0.038$. As shown in Fig. 4, in women AI correlated negatively with handedness, $r=-0.13$, but positively with footedness, $r=0.19$, $p \mathrm{~s}>0.10$, whereas in men AI correlated positively with handedness, $r=0.31, p=0.044$, but negatively with footedness, $r=-0.20, p=0.20$.

Turning Bias and Additional Analyses No significant effects pertaining to Schultheiss's hypothesis emerged for turning bias as dependent variable or when we replaced nPower (or $\mathrm{n}$ Affiliation) with nAchievement in the previously described analyses $(p s>0.05)$.

\section{Experimental Hand-Contraction Effects}

To examine effects of unilateral or bilateral hand contractions on changes in PSE raw motive and raw AI scores, we conducted a series of ANCOVAs in which we included the respective baseline PSE raw-score measure as a covariate and tested for main and interaction effects of left-hand and right-hand contractions.

Motive Measures For the three motive measures, results can be summarized as follows: First, main effects of hand (left, right) on motive changes were all nonsignificant, $p s>0.37$, which is inconsistent both with McClelland's (1986) hypothesis and Kuhl and Kazen's (2008) hypothesis. Second, at the descriptive level we observed a pattern of increased motive scores for nPower and nAchievement for unilateral, but not bilateral or no hand contractions (this constituted a marginally significant 


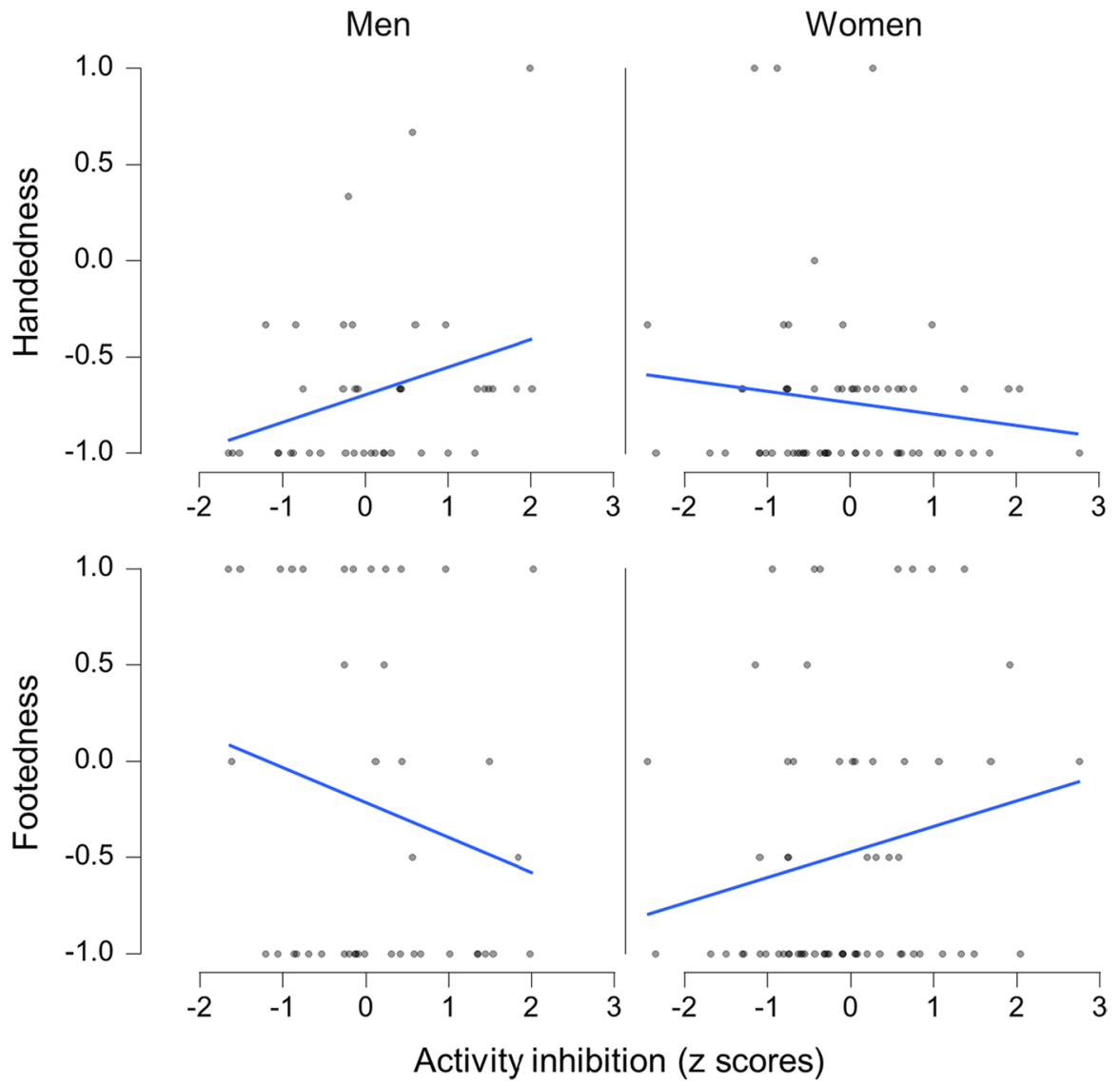

Fig. 4 Association between activity inhibition and self-reported handedness and footedness in men and women. Higher/positive values indicate greater leftward preference (right hemisphere), lower/negative values indicate greater rightward preference (left hemisphere). Figure available at https://osf.io/84usb/, under a CC-BY4.0 license

Left Hand x Right Hand effect for nPower, $\left.F(1,101)=3.64, p=0.059, \eta^{2}=0.035\right)$. For nAffiliation, we observed the reverse pattern, with decreasing nAffiliation scores for unilateral or bilateral hand contractions. We therefore followed an analytic strategy used in previous motive research (e.g., Brunstein et al., 1998) and aggregated motives separately for baseline and post-manipulation scores by subtracting nAffiliation scores from averaged nPower and nAchievement scores. For the resulting predominant motive disposition (PMD) scores, we obtained a significant Left Hand x Right Hand interaction, $F(1,101)=6.76, p=0.011, \eta^{2}=0.063$. As Fig. 5 shows, participants wrote stories with more achievement and power themes relative to affiliation themes after they had contracted either the left or the right hand. In contrast, they wrote stories with fewer achievement and power themes relative to affiliation themes after they had squeezed rubber balls with both hands or neither hand. None of the between-group differences became significant in post-hoc tests, $p s>0.18$. 
Fig. 5 Left hand $x$ right hand interaction effect on predominant motive disposition (PMD: [nPower + nAchievement $] / 2$ - nAffiliation, based on motive raw scores), adjusted for baseline PMD. Error bars represent SEM. Figure available at https://osf.io/84usb/, under a CC-BY4.0 license

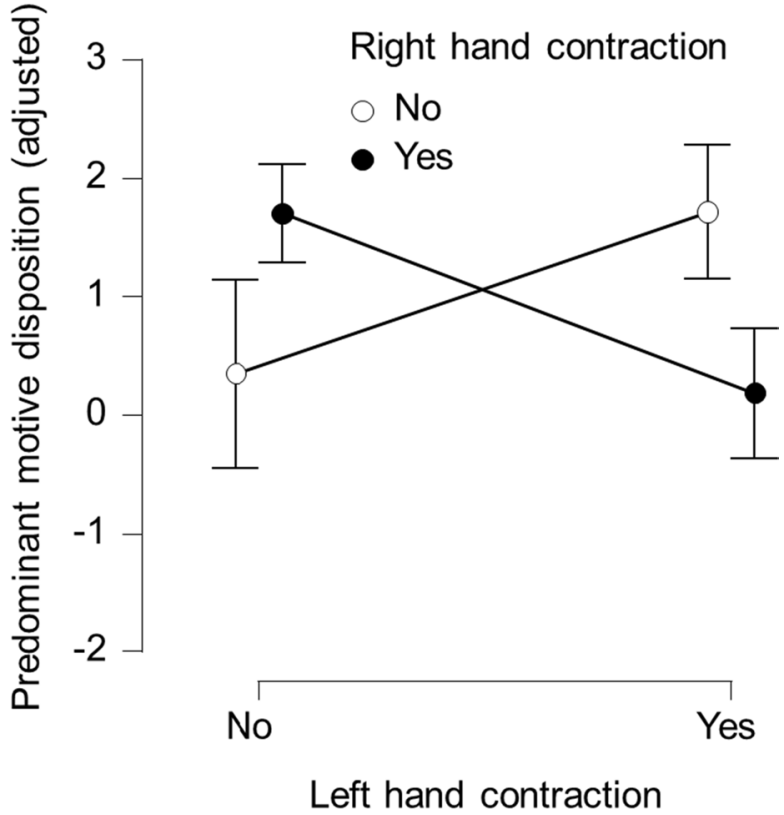

Al For AI score changes, we observed reductions resulting from left or right hand contractions at the descriptive level (see Fig. 6). In the case of left-hand contractions, these were significant, $F(1,102)=7.84, p=0.006$, partial $\eta^{2}=0.071$, but not in the case of right-hand contractions, $F(1,102)=0.53, p=0.47$, partial $\eta^{2}=0.005$. Left and right hand contractions had no significant interactive effect on AI changes, and neither did participant sex interact significantly with left-hand or right-hand contractions, $p s>0.12$.

In addition, we ran ANCOVAs to check whether overall word count was affected by experimental manipulations, but without significant results at the main effect level, $F_{\mathrm{S}}(1,102)<1, p \mathrm{~s}>0.34$, or for the left hand $\mathrm{x}$ right hand interaction, $F(1$, $101)=0.16, p=0.69$.

\section{Discussion}

Our main goal in this study was to explore the relationship between motive dispositions, including AI as a moderator of motives, and FCA measures, guided by hypotheses originally formulated by McClelland (1986; motives are represented in the RH), Kuhl and Kazen (2008; nPower is represented in the LH, nAffiliation in the RH), and Schultheiss (2018; motive-FCA associations depend on AI, with higher AI being associated with stronger $\mathrm{RH}$ than $\mathrm{LH}$ involvement). We also tested these hypotheses through the effects of left-hand and right-hand contractions on changes in motives and AI. Our findings can be summarized as follows: 
Fig. 6 Effects of left hand and right hand contractions on activity inhibition (raw scores), adjusted for baseline activity inhibition raw scores. Error bars represent SEM. Figure available at https://osf.io/84usb/, under a CC-BY4.0 license

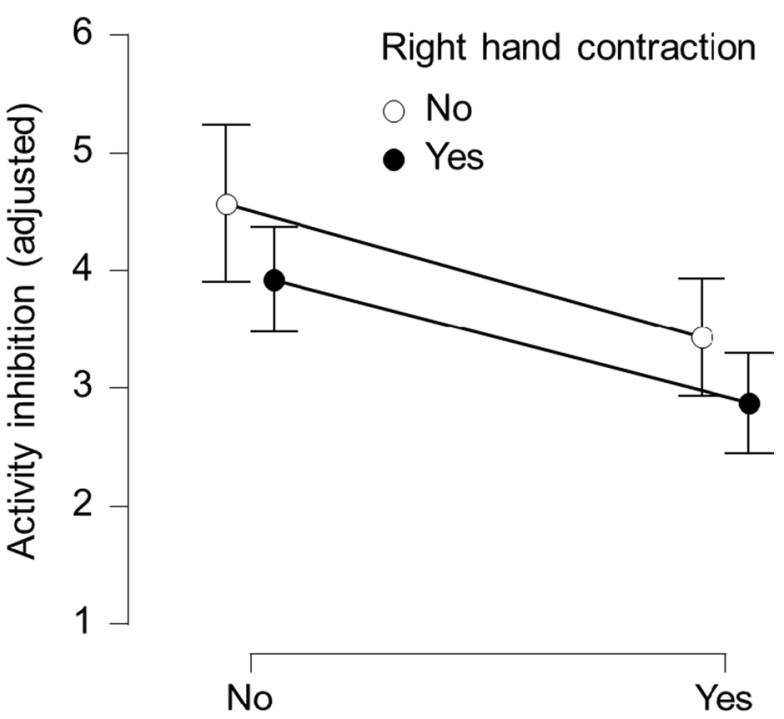

Left hand contraction

Looking at both associations between dispositional motives and FCAs and experimental manipulation effects on motive changes, we failed to find support for McClelland's or Kuhl and Kazen's hypotheses. Motives were not systematically associated with laterality measures in a way that suggested RH primacy. Although nPower tended to be associated with the CFT in a manner suggesting more $\mathrm{RH}$ involvement in men, this was not the case for women, and the pattern was not observed in Schütz and Schultheiss's (2020) earlier study. Perhaps by chance, higher average motives were even associated with an overall more rightward preference and thus LH involvement in terms of hand, foot, ear, and eye use. Left-hand contractions by themselves did not lead to motive increases, as would be expected based on McClelland's (1986) RH hypothesis. Likewise, there was little evidence for an association between nPower and greater LH dominance or nAffiliation and greater $\mathrm{RH}$ dominance for any of the laterality measures or for the idea that left-hand contractions increase nAffiliation and right-hand contractions increase nPower. Thus, we found no support for the Kuhl and Kazen (2008) hypothesis.

In contrast, several findings corroborated Schultheiss's (2018) conjecture that AI moderates the association between motives and laterality, although the findings were most pronounced for nPower, with some additional observations for nAffiliation, and no associations found for $\mathrm{nAchievement.} \mathrm{If} \mathrm{AI}$ was high, nPower was associated with more pseudoneglect (i.e., RH engagement) when participants bisected lines presented in the middle of the screen and with more efficient intrahemispheric than interhemispheric processing, as reflected in higher CUD scores. Conversely, in low-AI individuals, nPower was associated with more neglect (i.e., LH engagement) on the LBT, more typical rightward side preferences for hand, foot, eye, and ear use, and enhanced interhemispheric processing, as reflected in lower CUD scores. Thus, for nPower Schultheiss's hypothesis that motives are preferentially expressed through LH functions if $\mathrm{AI}$ is low and through $\mathrm{RH}$ functions if $\mathrm{AI}$ is high receives some support. 
For nAffiliation, fewer significant findings emerged and in the opposite direction of those we obtained for nPower: for limb preference (handedness and footedness), this motive was associated with a rightward side preference in high-AI individuals, but not in low-AI individuals, and on the Poffenberger task, nAffiliation was associated with enhanced interhemispheric transfer in high-AI individuals only. If taken at face value, these findings suggest that although nAffiliation tends to be less consistently associated with lateralized functions, the moderating effect of AI is in the opposite direction of Schultheiss's hypothesis of high AI being associated with more $\mathrm{RH}$, and low AI with more LH engagement, although it is consistent with the idea of a moderating effect of AI more generally. We also note that the opposite patterns of nPower x AI and nAffiliation x AI effects on laterality measures resonate to some extent with Kuhl and Kazen's hypothesis of differential associations of these motives with the LH and the RH, but in a more complex fashion than originally proposed.

Notably, however, the previously described conjoint effects of dispositional motives and AI emerged for men, but not for women, as reflected in a pervasive Motive x AI x Sex interaction pattern similar to the patterns observed in earlier studies on lateralized morphological markers of steroid exposure (Köllner \& Bleck, 2020; Schultheiss et al., 2019). We suspect that this may be due to at least two factors. First, although AI has been portrayed in earlier research as a latent disposition for RH engagement in attention tasks regardless of sex (Schultheiss et al., 2009), we found AI to be associated in different directions with handedness and footedness in men and women. In men, AI was generally linked to more left-handedness, but also more right-footedness, whereas in women we observed the opposite pattern of associations. This suggests that what may be true of attentional biases, for which Schultheiss et al. (2009) reported an overall AI-associated rightward attentional FCA in women and men alike, may not hold for other indicators of laterality, for which sex differences in AI emerge.

Second, previous research suggests that in women FCAs (e.g., Hausmann \& Burt, 2019), interhemispheric communication (e.g., Hausmann \& Güntürkün, 2000), and motivational needs (Schultheiss et al., 2003) vary across the menstrual cycle, and that these effects are different or absent in women using hormonal contraceptives. Thus, in contrast to men, whose hormone levels are affected primarily by a circadian rhythm and tend to be more stable over time (e.g., Liening et al., 2010), women experience more complex variations in their hormonal milieu over time and also through their use of hormonal contraceptives. Controlling for these factors and/or modelling them in research may be critical for determining if and when during the cycle associations between motives, AI, and FCAs emerge. Therefore, future studies should focus on naturally cycling women and assess salivary hormone levels to examine effects of estradiol, progesterone, and testosterone on concurrent task performance and/or motives across the menstrual cycle (e.g., Schultheiss \& Zimni, 2015 ) or test women in the menstrual phase only when levels of estradiol and progesterone are comparable to those of men and functional hemispheric asymmetries are more likely to be observed (e.g., Rode et al., 1995).

Note, however, that although we expect Motive $\mathrm{x}$ AI interactions to emerge more clearly in women under these conditions, we do not assume that they will necessarily resemble those we obtained for men. The main reasons for this caveat are the 
profound differential associations between sex and FCAs documented throughout neuropsychology in general (e.g., Cutting, 1997; Lezak et al., 2012; Ocklenburg \& Güntürkün, 2018) and, consistent with this but more specifically, the recently observed robust sex-dimorphic associations between AI and motive measures on the one hand and lateralized morphological markers of organizing effects of prenatal (Schultheiss et al., 2019) and pubertal steroid changes (Köllner \& Bleck, 2020) on the other. The latter observation suggests differential developmental trajectories of central nervous system lateralization patterns in women and men, which likely interact with activating effects of current fluctuations in steroid hormone concentrations in shaping lateralized cognitive and behavioral outcomes (see Ocklenburg \& Güntürkün, 2018, pp. 306 \& 307). The validity of this scenario can only be determined in future research that combines the study of lateralized brain functions with the measurement of motives and AI and the assessment of markers of organizing effects of sex steroids and current hormone concentrations in women and men.

Why did we observe motive-laterality associations more frequently for nPower than for nAffiliation and not at all for nAchievement? One possibility is that effects for nAffiliation and nAchievement are more subtle than those for nPower and require higher test power to be detected. While this may be true, we also think that there is considerable evidence now that nPower in particular is intimately tied to organizing (see above) and activating effects of gonadal steroids (Schultheiss, 2007; Stanton \& Schultheiss, 2009) and that because these hormones impinge both on the development and expression of this motive and FCAs, associations with laterality measures are more likely to be observed for nPower than for other motives. A third possible explanation concerns motive assessment. Winter's (1994) running-text system, which we employed for motive assessment in the present study, is particularly fine-grained and broadly validated for the assessment of nPower, but less so for nAffiliation and nAchievement. Perhaps the use of more nuanced coding systems for these motive dispositions, like McKay's (1992) affiliative trust/mistrust coding system or McClelland et al.'s (1953) original nAchievement coding system, will provide more clear-cut findings in future research.

Like our findings for dispositional motives, results for the hand-contraction manipulation suggest that motivational states can be changed through either LH or RH functional activation (but perhaps not both simultaneously). Participants who had repeatedly pressed a ball with either the left hand or the right hand subsequently wrote stories more saturated with power and achievement imagery and comparatively less saturated with affiliation imagery, whereas participants who either had not squeezed a ball or squeezed balls with both hands simultaneously wrote stories with less power and achievement imagery and relatively more affiliation imagery. These findings emerged above and beyond dispositional motive differences, which we controlled for in our analyses. However, the Left Hand x Right Hand interaction pattern was statistically significant only after combining motive measures into a PMD variable that contrasts the agentic motives nPower and nAchievement against the communal nAffiliation motive (see Brunstein et al., 1998). When we looked at each motive separately, the interaction effect was close to the 0.05 threshold for nPower only, whereas it failed to become significant for the other two motives. Moreover, probably due to the disordinal interaction pattern we were unable to find significant between-group differences in post-hoc analyses. We tentatively interpret our findings 
in the following way: if neither hemisphere is engaged in vigorous motor activity, a being-oriented affiliation-motivated state is likely to predominate (see McAdams \& Powers, 1981). However, if motor activation occurs in either the left or the right hemisphere, a doing-oriented power-motivated state (and to some extent an achievementmotivated state, too) takes hold (see Kuhl \& Kazen, 2008). If we take our results for bimanual activation at face value, they suggest that activation of motor areas in both hemispheres simultaneously leads to partial suppression of agentic motives, perhaps via interhemispheric inhibition processes. However, this interpretation rests on the assumption that the decreased agentic motivational state we observed in the bimanual squeezing condition can be replicated in future research using samples with better power for detecting conjoint effects of left- and right-hand contractions.

With regard to motor activation effects on AI we obtained a clear-cut asymmetry result: left-hand contractions, but not right-hand contractions, led to an AI decrease, with no sex difference evident for this effect. This observation is consistent with the characterization of AI as a lateralized attentional disposition (Schultheiss et al., 2009) and as a brake on action execution (McClelland, 1987), although the direction of the effect may be surprising at first blush: Why is activation of RH motor cortex associated with decreased AI? We offer the following explanation: The right prefrontal cortex has been described as a hub of inhibitory functions (Depue et al., 2016) and as the locus of sentential negations (Beltrán et al., 2018). Both descriptions fit the cognitive and behavioral correlates of AI. We therefore suspect that activation of the RH motor cortex by squeezing a ball with the left hand leads to a decrease of ipsilateral right prefrontal functions involved in inhibition and hence in the spontaneous use of the negation not, as reflected in reduced AI levels. This account is consistent with the observation that hand movements similar to those we used in our research are associated not only with the expected motor cortex signal increases, but also with signal decreases in ipsilateral prefrontal areas in an fMRI study (Nakata et al., 2019) and, in the case of left-hand contractions, with decreased cortical activity in an EEG study (Cross-Villasana et al., 2016). ${ }^{3}$

Results for experimental hand contraction manipulations on motives and AI could not be attributed to effects on overall word count, which stayed highly stable from baseline to post-manipulation assessment and changes in which failed to show significant main and interaction effects of the experimental manipulation.

\section{Limitations}

Although our present study advances our understanding of the links between motives and FCAs, we acknowledge a number of limitations associated with it beyond those we have already mentioned. First, the selection of FCA-associated tasks we used in the present research was biased towards the nonverbal-spatial domain; complementary tasks assessing functional asymmetries related to LH functions such as language (e.g., Willemin et al., 2016) and categorization (e.g., Gilbert et al., 2008) were missing from our battery.

\footnotetext{
3 As one reviewer suggested, future research could also employ the reverse strategy and test how experimental motive arousal (e.g., Schultheiss et al., 2004) impacts FCAs.
} 
Second, with the exception of turning bias and perhaps the motor laterality index derived from the Poffenberger task, one could argue that the FCA measures we employed were better geared towards assessing perceptual and cognitive laterality biases, but less well suited for measuring laterality of actual behavior operating on the environment, such as throwing, catching, or fine-motor performance. Future research on motives and laterality should therefore use a more comprehensive set of tests including, in addition to tests tapping nonverbal-spatial cognition, also tests assessing LH-lateralized cognitive functions as well as FCAs in behavioral performance (see, for instance, Parker et al., 2020).

Third, because we used brief and economical, but non-standard measures of handedness, footedness, and eye and ear preferences, our present findings may be difficult to compare to research employing more extensive, standardized measures such as the Edinburgh Handedness Inventory (Oldfield, 1971) or the Waterloo Footedness Questionnaire (Elias et al., 1998). Moreover, eye preference can also be assessed more directly and unobtrusively by using the hole-in-card test of ocular dominance (Miles, 1930) and ear preference via dichotic-listening tasks (Hugdahl, 2011).

Fourth, the CFT we employed featured only the emotion happiness. It might be worthwhile to use chimeric faces displaying multiple emotions in future research, particularly because motives are known to influence responses to facial expressions in specific ways (for review: Stanton et al., 2010) that may also interact with the side of presentation.

Fifth, although the four-picture PSE we used for the assessment of dispositional motives and AI provided reasonable score distributions, it was geared to the needs of this study and did not represent the more commonly used six-picture PSE described by Pang and Schultheiss (2005) for the assessment of motive dispositions. Here, too, future research could benefit from preferring the standardized instrument over custom-tailored assessment tools.

Finally, given the complexity of the Motive x AI x Sex interaction effects we observed in the present study, future studies should be better powered to detect such subtle effects, particularly when following them up for each sex. Although our sample was adequately powered to detect bivariate associations between motives and FCAs and thus to test the McClelland and Kuhl and Kazen hypotheses, it was underpowered to detect more complex two-way and three-way interactions and to provide adequate power for within-gender follow-up tests. Our previous arguments for cycle- and contraception-related effects notwithstanding, higher relative test power may help detect whatever associations between motives and FCAs may exist in women and men.

\section{Conclusion}

To sum up, the present research explored to what extent fundamental motivational needs for power, achievement, and affiliation are associated with FCAs, as assessed per tasks and self-report. Overall, results for motive dispositions were much more consistent with Schultheiss's (2018) hypothesis that motives are associated with 
functional hemispheric asymmetries (and interhemispheric processing) in an AIdependent fashion than either with McClelland's (1986) RH hypothesis or with Kuhl and Kazen's (2008) nPower-LH/nAffiliation-RH hypothesis. However, they emerged in a sex-dimorphic way and were most frequent for nPower, limited for nAffiliation, and absent for nAchievement. Experimental manipulation of LH and RH motor cortex activation through right-hand and left-hand contractions, respectively, likewise suggested that both hemispheres could contribute to changes in motivational states, particularly with regard to power motivation. In contrast, changes in AI were specifically associated with RH activation. Overall, these findings suggest that more in-depth research on the link between motives and dispositional laterality (AI) on the one hand and FCA measures on the other has great potential for advancing both motivation science and the study of laterality.

Acknowledgements We thank Kevin Janson for his support.

Funding Open Access funding enabled and organized by Projekt DEAL.

\section{Declarations}

Ethical approval The procedures performed in this study were approved by the local institutional research committee and in accordance with the 1964 Helsinki declaration and its later amendments.

Informed consent Informed consent was obtained in writing from all individual participants included in the study.

Conflict of interest Oliver Schultheiss, Olivia Schwemmer, and Ksenia Khalaidovski declare that they have no conflict of interest to disclose.

Open Access This article is licensed under a Creative Commons Attribution 4.0 International License, which permits use, sharing, adaptation, distribution and reproduction in any medium or format, as long as you give appropriate credit to the original author(s) and the source, provide a link to the Creative Commons licence, and indicate if changes were made. The images or other third party material in this article are included in the article's Creative Commons licence, unless indicated otherwise in a credit line to the material. If material is not included in the article's Creative Commons licence and your intended use is not permitted by statutory regulation or exceeds the permitted use, you will need to obtain permission directly from the copyright holder. To view a copy of this licence, visit http://creativecommons.org/licen ses/by/4.0/.

\section{References}

Atkinson, J. W. (1957). Motivational determinants of risk-taking behavior. Psychological Review, 64, 359-372. https://doi.org/10.1037/h0043445.

Beckmann, J., Gröpel, P., \& Ehrlenspiel, F. (2013). Preventing motor skill failure through hemispherespecific priming: Cases from choking under pressure. Journal of Experimental Psychology: General, 142(3), 679-691. https://doi.org/10.1037/a0029852.

Beltrán, D., Muñetón-Ayala, M., \& de Vega, M. (2018). Sentential negation modulates inhibition in a stop-signal task. Evidence from behavioral and ERP data. Neuropsychologia, 112, 10-18. https:// doi.org/10.1016/j.neuropsychologia.2018.03.004.

Berlucchi, G., Heron, W., Hyman, R., Rizzolatti, G., \& Umilta, C. (1971). Simple reaction times of ipsilateral and contralateral hand to lateralized visual stimuli. Brain, 94(3), 419-430. https://doi.org/10. 1093/brain/94.3.419. 
Borchert, K. (2013). Poffenberger paradigm task. In Millisecond Software, LLC. https://www.millisecond.com/download/library/poffenberger/.

Borsboom, D., Mellenbergh, G. J., \& van Heerden, J. (2004). The concept of validity. Psychological Review, 111(4), 1061-1071. https://doi.org/10.1037/0033-295X.111.4.1061.

Brunstein, J. C., Schultheiss, O. C., \& Grässmann, R. (1998). Personal goals and emotional well-being: The moderating role of motive dispositions. Journal of Personality and Social Psychology, 75(2), 494-508. https://doi.org/10.1037/0022-3514.75.2.494.

Cross-Villasana, F., Gröpel, P., Doppelmayr, M., \& Beckmann, J. (2016). Unilateral left-hand contractions produce widespread depression of cortical activity after their execution. PLoS ONE, 10(12), e0145867. https://doi.org/10.1371/journal.pone.0145867.

Cutting, J. (1997). Principles of psychopathology. Two worlds -- two minds -- two hemispheres. Oxford University Press.

Depue, B. E., Orr, J. M., Smolker, H. R., Naaz, F., \& Banich, M. T. (2016). The organization of right prefrontal networks reveals common mechanisms of inhibitory regulation across cognitive, emotional, and motor processes. Cerebral Cortex, 26(4), 1634-1646. https://doi.org/10.1093/cercor/bhu324.

Dimond, S. J., \& Farrington, L. (1977). Emotional response to films shown to the right or left hemisphere of the brain measured by heart rate. Acta Psychologica, 41(4), 255-260. https://doi.org/10.1016/ 0001-6918(77)90020-8.

Drescher, A., \& Schultheiss, O. C. (2016). Meta-analytic evidence for higher implicit affiliation and intimacy motivation scores in women, compared to men. Journal of Research in Personality, 64, 1-10. https://doi.org/10.1016/j.jrp.2016.06.019.

Elias, L. J., Bryden, M. P., \& Bulman-Fleming, M. B. (1998). Footedness is a better predictor than is handedness of emotional lateralization. Neuropsychologia, 36(1), 37-43. https://doi.org/10.1016/ S0028-3932(97)00107-3.

Frisch, M., \& Schultheiss, O. C. (2012). PSECoder software. Author.

Gainotti, G. (2012). Unconscious processing of emotions and the right hemisphere. Neuropsychologia, 50(2), 205-218. https://doi.org/10.1016/j.neuropsychologia.2011.12.005.

Gazzaniga, M. S. (1985). The social brain: Discovering the networks of the mind. Basic Books.

Gilbert, A. L., Regier, T., Kay, P., \& Ivry, R. B. (2008). Support for lateralization of the Whorf effect beyond the realm of color discrimination. Brain and Language, 105(2), 91-98. https://doi.org/10. 1016/j.bandl.2007.06.001.

Harmon-Jones, E. (2006). Unilateral right-hand contractions cause contralateral alpha power suppression and approach motivational affective experience. Psychophysiology, 43(6), 598-603. https://doi.org/ 10.1111/j.1469-8986.2006.00465.x.

Hausmann, M., \& Burt, D. M. (2019). Sex hormonal effects on brain lateralization. In O. C. Schultheiss \& P. H. Mehta (Eds.), Routledge international handbook of social neuroendocrinology. (pp. 357370). Routledge. https://doi.org/10.4324/9781315200439-21.

Hausmann, M., Ergun, G., Yazgan, Y., \& Güntürkün, O. (2002). Sex differences in line bisection as a function of hand. Neuropsychologia, 40(3), 235-240. https://doi.org/10.1016/s0028-3932(01) 00112-9.

Hausmann, M., \& Güntürkün, O. (2000). Steroid fluctuations modify functional cerebral asymmetries: the hypothesis of progesterone-mediated interhemispheric decoupling. Neuropsychologia, 38(10), 1362-1374. https://doi.org/10.1016/S0028-3932(00)00045-2.

Hellige, J. B. (1993). Hemispheric asymmetry. What's right and what's left. Harvard University Press.

Hofer, J., Busch, H., Bender, M., Ming, L., \& Hagemeyer, B. (2010). Arousal of achievement motivation among student samples in three different cultural contexts: Self and social standards of evaluation. Journal of Cross-Cultural Psychology, 41, 758-775. https://doi.org/10.1177/0022022110375160.

Hugdahl, K. (2011). Fifty years of dichotic listening research - still going and going and... Brain and Cognition, 76(2), 211-213. https://doi.org/10.1016/j.bandc.2011.03.006.

Innes, B. R., Burt, D. M., Birch, Y. K., \& Hausmann, M. (2016). A leftward bias however you look at it: Revisiting the emotional chimeric face task as a tool for measuring emotion lateralization. Laterality, 21(4-6), 643-661. https://doi.org/10.1080/1357650X.2015.1117095.

Janson, K. T., Bleck, K., Fenkl, J., Riegl, L. T., Jägel, F., \& Köllner, M. G. (2018). Inhibited power motivation is associated with the facial width-to-height ratio in females. Adaptive Human Behavior and Physiology, 4(1), 21-41. https://doi.org/10.1007/s40750-017-0075-y.

Jemmott, J. B., Borysenko, J. Z., Borysenko, M., McClelland, D. C., Chapman, R., Meyer, D., \& Benson, H. (1983). Academic stress, power motivation, and decrease in secretion rate of salivary secretory immunoglobulin A. Lancet, 8339, 1400-1402. https://doi.org/10.1016/S0140-6736(83)92354-1. 
Jewell, G., \& McCourt, M. E. (2000). Pseudoneglect: a review and meta-analysis of performance factors in line bisection tasks. Neuropsychologia, 38(1), 93-110. https://doi.org/10.1016/S0028-3932(99) 00045-7.

Koestner, R., \& McClelland, D. C. (1992). The affiliation motive. In C. P. Smith (Ed.), Motivation and personality: Handbook of thematic content analysis. (pp. 205-210). Cambridge University Press.

Köllner, M., \& Schultheiss, O. C. (2014). Meta-analytic evidence of low convergence between implicit and explicit measures of the needs for achievement, affiliation, and power. Frontiers in Psychology, 5, 826. https://doi.org/10.3389/fpsyg.2014.00826.

Köllner, M. G., \& Bleck, K. (2020). Exploratory evidence of sex-dimorphic associations of the ulnato-fibula ratio, a potential marker of pubertal sex steroid exposure, with the implicit need for power. Adaptive Human Behavior and Physiology, 6(1), 93-118. https://doi.org/10.1007/ s40750-020-00130-8.

Köllner, M. G., Janson, K. T., \& Bleck, K. (2019). The social biopsychology of implicit motive development. In O. C. Schultheiss \& P. H. Mehta (Eds.), Routledge international handbook of social neuroendocrinology. (pp. 568-585). Routledge. https://doi.org/10.4324/9781315200439-32.

Kuhl, J., \& Kazen, M. (2008). Motivation, affect, and hemispheric asymmetry: power versus affiliation. Journal of Personality and Social Psychology, 95(2), 456-469. https://doi.org/10.1037/0022-3514. 95.2.456.

Kuper, N., Käckenmester, W., \& Wacker, J. (2019). Resting frontal EEG asymmetry and personality traits: A meta-analysis. European Journal of Personality. https://doi.org/10.1002/per.2197.

Lang, C. (2016). Effekte der Temperatur auf die impliziten Motive [Ambient-temperature effects on implicit motives]. Friedrich-Alexander University.

Levy, J., Heller, W., Banich, M. T., \& Burton, L. A. (1983). Asymmetry of perception in free viewing of chimeric faces. Brain and Cognition, 2(4), 404-419. https://doi.org/10.1016/0278-2626(83) 90021-0.

Lezak, M. D., Howieson, D. B., Bigler, E. D., \& Tranel, D. (2012). Neuropsychological assessment. (5th ed.). Oxford University Press.

Liening, S. H., Stanton, S. J., Saini, E. K., \& Schultheiss, O. C. (2010). Salivary testosterone, cortisol, and progesterone: two-week stability, interhormone correlations, and effects of time of day, menstrual cycle, and oral contraceptive use on steroid hormone levels. Physiology and Behavior, 99(1), 8-16. https://doi.org/10.1016/j.physbeh.2009.10.001.

Marzi, C. A., Bisiacchi, P., \& Nicoletti, R. (1991). Is interhemispheric transfer of visuomotor information asymmetric? Evidence from a meta-analysis. Neuropsychologia, 29(12), 1163-1177. https://doi.org/ 10.1016/0028-3932(91)90031-3.

Mason, A., \& Blankenship, V. (1987). Power and affiliation motivation, stress, and abuse in intimate relationships. Journal of Personality and Social Psychology, 52, 203-210. https://doi.org/10.1037/00223514.52.1.203.

McAdams, D. P., \& Powers, J. (1981). Themes of intimacy in behavior and thought. Journal of Personality and Social Psychology, 40, 573-587. https://doi.org/10.1037/0022-3514.40.3.573.

McClelland, D. C. (1958). Methods of measuring human motivation. In J. W. Atkinson (Ed.), Motives in fantasy, action, and society: A method of assessment and study. (pp. 7-42). Van Nostrand.

McClelland, D. C. (1979). Inhibited power motivation and high blood pressure in men. Journal of Abnormal Psychology, 88, 182-190. https://doi.org/10.1037/0021-843X.88.2.182.

McClelland, D. C. (1986). Some reflections on the two psychologies of love. Journal of Personality, 54, 334-353. https://doi.org/10.1111/j.1467-6494.1986.tb00398.x.

McClelland, D. C. (1987). Human motivation. Cambridge University Press.

McClelland, D. C. (1989). Motivational factors in health and disease. American Psychologist, 44, 675683. https://doi.org/10.1037/0003-066X.44.4.675.

McClelland, D. C., Atkinson, J. W., Clark, R. A., \& Lowell, E. L. (1953). The achievement motive. Appleton-Century-Crofts.

McClelland, D. C., \& Boyatzis, R. E. (1982). Leadership motive pattern and long-term success in management. Journal of Applied Psychology, 67, 737-743. https://doi.org/10.1037/0021-9010.67.6.737.

McClelland, D. C., \& Burnham, D. H. (2003). Power is the great motivator. Harvard Business Review, $81(1), 117-126+142$.

McClelland, D. C., Koestner, R., \& Weinberger, J. (1989). How do self-attributed and implicit motives differ? Psychological Review, 96, 690-702. https://doi.org/10.1037/0033-295X.96.4.690.

McGilchrist, I. (2009). The master and his emissary. Yale University Press. 
McKay, J. R. (1992). A scoring system for affiliative trust-mistrust. In C. P. Smith (Ed.), Motivation and personality: Handbook of thematic content analysis. (pp. 266-277). Cambridge University Press.

McManus, I. C., Porac, C., Bryden, M. P., \& Boucher, R. (1999). Eye-dominance, writing hand, and throwing hand. Laterality, 4(2), 173-192. https://doi.org/10.1080/713754334.

Miles, W. (1930). Ocular dominance in human adults. Journal of General Psychology, 4, 412. https:// doi.org/10.1080/00221309.1930.9918218.

Nakata, H., Domoto, R., Mizuguchi, N., Sakamoto, K., \& Kanosue, K. (2019). Negative BOLD responses during hand and foot movements: An fMRI study. PLoS ONE, 14(4), e0215736. https://doi.org/10.1371/journal.pone.0215736.

Ocklenburg, S., \& Güntürkün, O. (2018). The lateralized brain. The neuroscience and evolution of hemispheric asymmetries. Academic. https://doi.org/10.1016/C2014-0-03755-0.

Oldfield, R. C. (1971). The assessment and analysis of handedness: The Edinburgh inventory. Neuropsychologia, 9(1), 97-113. https://doi.org/10.1016/0028-3932(71)90067-4.

Pang, J. S., \& Schultheiss, O. C. (2005). Assessing implicit motives in U.S. College students: Effects of picture type and position, gender and ethnicity, and cross-cultural comparisons. Journal of Personality Assessment, 85(3), 280-294. https://doi.org/10.1207/s15327752jpa8503_04.

Parker, A. J., Woodhead, Z. V. J., Thompson, P. A., \& Bishop, D. V. M. (2020). Assessing the reliability of an online behavioural laterality battery: A pre-registered study. Laterality, 1-39.https://doi. org/10.1080/1357650X.2020.1859526.

Poffenberger, A. (1912). Reaction time to retinal stimulation with special reference to the time lost in conduction through nervous centers. Archives of Psychology, 23, 1-73.

Rode, C., Wagner, M., \& Güntürkün, O. (1995). Menstrual cycle affects functional cerebral asymmetries. Neuropsychologia, 33(7), 855-865. https://doi.org/10.1016/0028-3932(95)00015-U.

Rösch, A. G., Stanton, S. J., \& Schultheiss, O. C. (2013). Implicit motives predict affective responses to emotional expressions. Frontiers in Psychology, 4, 985. https://doi.org/10.3389/fpsyg.2013. 00985.

Saron, C. D., Foxe, J. J., Schroeder, C. E., \& Vaughan, H. G. (2003). Complexities of interhemispheric communication in sensorimotor tasks revealed by high-density event-related potential mapping. In K. Hugdahl \& R. J. Davidson (Eds.), The asymmetrical brain. (pp. 341-408). MIT Press.

Schiff, B. B., \& Lamon, M. (1994). Inducing emotion by unilateral contraction of hand muscles. Cortex, 30(2), 247-254. https://doi.org/10.1016/S0010-9452(13)80196-7.

Schönbrodt, F. D., Hagemeyer, B., Brandstätter, V., Czikmantori, T., Gröpel, P., Hennecke, M., Israel, L. S. F., Janson, K. T., Kemper, N., Köllner, M. G., Kopp, P. M., Mojzisch, A., Müller-Hotop, R., Prüfer, J., Quirin, M., Scheidemann, B., Schiestel, L., Schulz-Hardt, S., Sust, L. N. N., Zygar-Hoffmann, C., \& Schultheiss, O. C. (2020). Measuring implicit motives with the Picture Story Exercise (PSE): Databases of expert-coded German stories, pictures, and updated picture norms. Journal of Personality Assessment, 1-14.https://doi.org/10.1080/00223891.2020.1726936.

Schultheiss, O. C. (2007). A biobehavioral model of implicit power motivation arousal, reward and frustration. In E. Harmon-Jones \& P. Winkielman (Eds.), Social neuroscience: Integrating biological and psychological explanations of social behavior. (pp. 176-196). Guilford.

Schultheiss, O. C. (2018). Implicit motives and hemispheric processing differences are critical for understanding personality disorders: A Commentary on Hopwood. European Journal of Personality, 32(5), 580-582. https://doi.org/10.1002/per.2174.

Schultheiss, O. C., \& Brunstein, J. C. (2002). Inhibited power motivation and persuasive communication: A lens model analysis. Journal of Personality, 70, 553-582. https://doi.org/10.1111/1467-6494. 05014.

Schultheiss, O. C., Dargel, A., \& Rohde, W. (2003). Implicit motives and gonadal steroid hormones: Effects of menstrual cycle phase, oral contraceptive use, and relationship status. Hormones and Behavior, 43, 293-301. https://doi.org/10.1016/S0018-506X(03)00003-5.

Schultheiss, O. C., Frisch, M., Özbe, D., Ossmann, A., Schultheiss, M., Lentz, S., Martin, L., \& Rösch, A. G. (2019). Implicit motives show sex-dimorphic associations with digit ratio. Motivation Science, 5(4), 326-342. https://doi.org/10.1037/mot0000122.

Schultheiss, O. C., \& Köllner, M. G. (2021). Implicit motives. In O. P. John \& R. W. Robins (Eds.), Handbook of personality: Theory and research. (4th ed., pp. 385-410). Guilford.

Schultheiss, O. C., Köllner, M. G., Busch, H., \& Hofer, J. (2020). Evidence for a robust, estradiol-associated sex difference in narrative-writing fluency. Neuropsychology. https://doi.org/10.1037/neu00 00706. 
Schultheiss, O. C., \& Pang, J. S. (2007). Measuring implicit motives. In R. W. Robins, R. C. Fraley, \& R. Krueger (Eds.), Handbook of research methods in personality psychology. (pp. 322-344). Guilford.

Schultheiss, O. C., Patalakh, M., Rawolle, M., Liening, S., \& MacInnes, J. J. (2011). Referential competence is associated with motivational congruence. Journal of Research in Personality, 45, 59-70. https://doi.org/10.1016/j.jrp.2010.11.014.

Schultheiss, O. C., Riebel, K., \& Jones, N. M. (2009). Activity inhibition: A predictor of lateralized brain function during stress? Neuropsychology, 23, 392-404. https://doi.org/10.1037/a0014591.

Schultheiss, O. C., \& Rohde, W. (2002). Implicit power motivation predicts men's testosterone changes and implicit learning in a contest situation. Hormones and Behavior, 41, 195-202. https://doi.org/ 10.1006/hbeh.2001.1745.

Schultheiss, O. C., Wirth, M. M., \& Stanton, S. J. (2004). Effects of affiliation and power motivation arousal on salivary progesterone and testosterone. Hormones and Behavior, 46(5), 592-599. https:// doi.org/10.1016/j.yhbeh.2004.07.005.

Schultheiss, O. C., \& Zimni, M. (2015). Associations between implicit motives and salivary steroids, 2D:4D digit ratio, mental rotation performance, and verbal fluency. Adaptive Human Behavior and Physiology, 1(4), 387-407. https://doi.org/10.1007/s40750-014-0012-2.

Schütz, L.-M., \& Schultheiss, O. C. (2020). Implicit motives, laterality, sports participation and competition in gymnasts. Frontiers in Psychology, 11, 900. https://doi.org/10.3389/fpsyg.2020.00900.

Stanton, S. J., Hall, J. L., \& Schultheiss, O. C. (2010). Properties of motive-specific incentives. In O. C. Schultheiss \& J. C. Brunstein (Eds.), Implicit motives. (pp. 245-278). Oxford University Press.

Stanton, S. J., \& Schultheiss, O. C. (2009). The hormonal correlates of implicit power motivation. Journal of Research in Personality, 43, 942-949. https://doi.org/10.1016/j.jrp.2009.04.001.

Steinmann, B., Dörr, S. L., Schultheiss, O. C., \& Maier, G. W. (2015). Implicit motives and leadership effectiveness revisited: What constitutes the leadership motive pattern? Motivation and Emotion, 39, 167-174. https://doi.org/10.1007/s11031-014-9458-6.

Steinmann, B., Otting, S. K., \& Maier, G. W. (2016). Need for affiliation as a motivational add-on for leadership behaviors and managerial success. Frontiers in Psychology, 7, 1972. https://doi.org/10. 3389/fpsyg.2016.01972.

Thomas, C. L., Bourdeau, A. M., \& Tagler, M. J. (2018). Interhemispheric communication and the preference for attitude consistent information. Laterality: Asymmetries of Body, Brain and Cognition, 1-13. https://doi.org/10.1080/1357650X.2018.1520860.

Wiemers, U. S., Schultheiss, O. C., \& Wolf, O. T. (2015). Public speaking in front of an unreceptive audience increases implicit power motivation and its endocrine arousal signature. Hormones and Behavior, 71, 69-74. https://doi.org/10.1016/j.yhbeh.2015.04.007.

Willemin, J., Hausmann, M., Brysbaert, M., Dael, N., Chmetz, F., Fioravera, A., Gieruc, K., \& Mohr, C. (2016). Stability of right visual field advantage in an international lateralized lexical decision task irrespective of participants' sex, handedness or bilingualism. Laterality, 21(4-6), 502-524. https:// doi.org/10.1080/1357650X.2015.1130716.

Winter, D. G. (1973). The power motive. Free Press.

Winter, D. G. (1994). Manual for scoring motive imagery in running text (4 ed.). Unpublished manuscript.

Winter, D. G. (1998). The contributions of David McClelland to personality assessment. Journal of Personality Assessment, 71, 129-145. https://doi.org/10.1207/s15327752jpa7102_1.

Wittling, W. (1995). Brain asymmetry in the control of autonomic-physiologic activity. In R. J. Davidson \& K. Hugdahl (Eds.), Brain asymmetry. (pp. 305-357). MIT Press.

Yazgan, M. Y., Leckman, J. F., \& Wexler, B. E. (1996). A direct observational measure of whole body turning bias. Cortex, 32(1), 173-176. https://doi.org/10.1016/S0010-9452(96)80025-6.

Publisher's note Springer Nature remains neutral with regard to jurisdictional claims in published maps and institutional affiliations. 